\title{
ÉVOLUTION DES PEUPLEMENTS MAMMALIENS EN EUROPE DU NORD-OUEST DURANT LE PLÉISTOCÈNE MOYEN ET SUPÉRIEUR. LE CAS DE LA FRANCE SEPTENTRIONALE
}

Patrick Auguste

\section{To cite this version:}

Patrick Auguste. ÉVOLUTION DES PEUPLEMENTS MAMMALIENS EN EUROPE DU NORDOUEST DURANT LE PLÉISTOCÈNE MOYEN ET SUPÉRIEUR. LE CAS DE LA FRANCE SEPTENTRIONALE. Quaternaire, AFEQ-CNF INQUA, 2009, 20 (4), pp.527. hal-02110557

\section{HAL Id: hal-02110557 \\ https://hal.archives-ouvertes.fr/hal-02110557}

Submitted on 20 May 2019

HAL is a multi-disciplinary open access archive for the deposit and dissemination of scientific research documents, whether they are published or not. The documents may come from teaching and research institutions in France or abroad, or from public or private research centers.
L'archive ouverte pluridisciplinaire HAL, est destinée au dépôt et à la diffusion de documents scientifiques de niveau recherche, publiés ou non, émanant des établissements d'enseignement et de recherche français ou étrangers, des laboratoires publics ou privés. 


\section{ÉVOLUTION DES PEUPLEMENTS MAMMALIENS \\ EN EUROPE DU NORD-OUEST DURANT \\ LE PLÉISTOCÈNE MOYEN ET SUPÉRIEUR. \\ LE CAS DE LA FRANCE SEPTENTRIONALE}

\section{Patrick AUGUSTE}

\section{RÉSUMÉ}

La France septentrionale constitue durant le Pléistocène moyen et supérieur un carrefour biogéographique à l'échelle européenne où les influences directes des variations climatiques et environnementales cycliques sont particulièrement bien enregistrées. De nombreux gisements paléolithiques et paléontologiques ayant livré des vestiges de grande faune mammalienne jalonnent cette région depuis environ un million d'années jusqu'à la fin du Pléistocène. Les associations de grands mammifères identifiées présentent l'intérêt d'être bien datées et replacées dans un contexte paléoécologique cohérent. Cette présente synthèse est la première à être réalisée pour le Pléistocène moyen et supérieur en France septentrionale. Vingt-huit gisements ont été sélectionnés et soixante-huit taxons ont été pris en compte. Il est ainsi possible de suivre la dynamique de renouvellement des différents taxons durant cette période, mais également de proposer un scénario global permettant d'interpréter la récurrence des grandes associations fauniques analysées. Au moins huit événements majeurs de renouvellements de faune ont été identifiés et bien caractérisés et datés assez précisément. Ceux-ci surviennent soit au début, soit au cours des stades isotopiques $15,11,8,7 \mathrm{c}, 6,5 \mathrm{e}, 4 / 3 \mathrm{et} 2$. Ce sont surtout les herbivores qui sont les plus informatifs, ceux-ci étant directement tributaires des modifications de la végétation induites par les changements climatiques de faibles ou fortes amplitudes. Un impact régulateur des mégaherbivores et des animaux de taille plus modeste au sein des différents écosystèmes mis en évidence suivant les phases climatiques est envisagé, celui-ci s'inscrit bien dans le modèle du fonctionnement des systèmes complexes que constituaient les biomes pléistocènes. Le constat établi pour la France septentrionale s'insère parfaitement dans le cadre plus élargi de l'Europe du Nord-Ouest, mettant en exergue l'unité paléobiogéographique de cette région.

Mots-clés : Grands Mammifères, Pléistocène moyen, Pléistocène supérieur, France septentrionale, Europe du Nord-Ouest, biochronologie, Paléoécologie.

\section{ABSTRACT}

EVOLUTION OF THE MAMMAL SETTLEMENTS IN NORTH-WEST EUROPE DURING MIDDLE AND UPPER PLEISTOCENE: THE CASE OF NORTHERN FRANCE

Northern France is during the Middle and Upper Pleistocene a biogeographic crossroads at the European level where the direct influence of cyclic climatic and environmental variations is particularly well recorded. Many Palaeolithic and paleontological sites have yielded the remains of large mammals along this region for about a million years until the end of the Upper Pleistocene. Identified large mammals associations give the interest to be well dated and placed in a paleoecological background consistent. This synthesis is the first to be realized for the Middle and Upper Pleistocene in northern France. Twenty eight sites have been examined and sixty eight taxa used. It is thus possible to examine the renewal dynamics of the different taxa during this period, but also to propose a global model to interpret the recurrence of major faunal associations. At least eight major dispersal events have been highlighted, with good definitions and datations. They take place at the beginning or during the marine isotopic stage $15,11,8,7 \mathrm{c}, 6,5 \mathrm{e}, 4 / 3$ and 2. It is mainly the herbivorous that are most informative, since they are directly dependent on modifications in vegetation caused by low or high amplitudes climate changes. A regulatory impact of megaherbivorous and smaller animals in different ecosystems and different type of climate is considered, it well fits into the functioning model of complex systems that constituted the Pleistocene biomes. The interpretation for the northern France fits into the broader framework of the North-West Europe, emphasizing the paleobiogeographic unity of this region.

Keys-words: Large mammals, Middle Pleistocene, Upper Pleistocene, Northern France, North-West Europe, Biochronology, Paleoecology.

\section{1 - INTRODUCTION}

De manière générale, l'Europe du Nord-Ouest constitue durant le Pléistocène une entité paléobiogéographique cohérente où les dynamiques climatiques, environnementales et fauniques apparaissent assez homogènes. La France septentrionale, située au carrefour entre les Îles britanniques et le reste de l'Europe continentale, joue un rôle important au cours du Pléistocène moyen et supérieur en constituant une zone clé témoignant en particulier des renouvellements des grandes faunes mammaliennes. Par ailleurs, la position septentrionale de cette région lui impose des réponses plus rapides et bien plus marquées que ce qui s'observe par exemple dans les zones méditerranéennes.

Les contextes sédimentaires et géomorphologiques du Pléistocène moyen et supérieur identifiés dans le Nord de la France permettent, de par les approches pluridisci-

UMR CNRS 8157, Laboratoire Géosystèmes, Université Lille 1, bâtiment SN 5, 59655 Villeneuve d'Ascq cedex, France.

Courriel: patrick.auguste@univ-lille1.fr 
plinaires développées depuis de nombreuses années, de suivre avec une grande précision les modalités chronologiques de la dynamique des peuplements de grands mammifères dont les vestiges sont contenus au sein des séquences stratigraphiques (Antoine et al., 1998; Antoine et al., 2007). Le cadre paléoécologique est également bien renseigné, permettant de replacer les associations mammaliennes reconnues au sein d'une reconstitution des paléoécosystèmes cohérente (Antoine et al. 2003). Enfin, les témoignages des occupations humaines sont bien identifiés et permettent ainsi de mettre en parallèle l'évolution des peuplements humains avec la dynamique faunique (Tuffreau, 1987).

Les données publiées concernant les différentes successions des peuplements mammaliens dans le Nord de la France sont à ce jour soit cantonnées à des zones restreintes, comme la vallée de la Somme (Auguste, 1995b) ou la Normandie (Auguste, 2008), soit limitées à un gisement comme par exemple la Celle-sous-Moret (Limondin-Lozouet et al., 2006), Caours (Antoine et al., 2006) ou Biache-Saint-Vaast (Auguste, 1988). Le seul travail synthétique réalisé est demeuré inédit et date de plus d'une dizaine d'années (Auguste, 1995a). Récemment, un bilan rapide de ces successions fauniques identifiées au sein de gisements pléistocènes mais provenant uniquement de contexte fluviatile a été publié dans le cadre d'un article général (Auguste in Schreve et al., 2007).

Le but de cet article est de mettre à disposition les données actualisées et détaillées concernant les principales séries fauniques de grands mammifères provenant de 28 gisements permettant de retracer l'histoire de leurs peuplements au sein de la France septentrionale, depuis la fin du Pléistocène inférieur et le début du Pléistocène moyen jusqu'à l'extrême fin du Pléistocène supérieur. Cette synthèse sera ensuite confrontée avec les données provenant de zones limitrophes de la même grande entité géographique (Grande-Bretagne et Pays-Bas).

\section{2 - CADRE CHRONOSTRATIGRAPHIQUE}

Les gisements fossilifères ayant livré des vestiges de grands mammifères pléistocènes ne sont connus en France septentrionale qu'à partir de la fin du Pléistocène inférieur, après un million d'années. Ils sont relativement peu nombreux jusque vers 300000 ans BP, pour des raisons peut être taphonomiques. À partir de cette période, le nombre de gisements devient conséquent, en particulier durant les épisodes tempérés du complexe stratigraphique saalien (MIS 7) (fig. 1).

Il est à noter que dans presque tous les cas, les faunes sont associées avec des industries lithiques. Par ailleurs, de nombreux gisements paléolithiques n'ont pas livré de vestiges mammaliens, essentiellement à cause du contexte sédimentaire défavorable à la conservation de ces derniers, mais que la présence initiale de la faune lors de la formation du dépôt est toujours très probable (traces d'utilisation sur matières dures ou tendres animales sur les industries par exemple). C'est le cas en particulier pour le début glaciaire weichsélien (MIS $5 \mathrm{~d}$ à a) où très peu de séries fauniques ont pu être mises au jour de par la décarbonatation trop importante des sédiments encaissants ayant entraîné la disparition des vestiges (voir par exemple le cas de Bettencourt-SaintOuen: Auguste, 2002; Locht, 2002).

D'un point de vue biochronologique, toutes les faunes mammaliennes de France septentrionale s'inscrivent au sein des biozones ou standardzones définies par Guérin (1980), depuis le début de la biozone 20 jusqu'à la fin de la 26. Les équivalents chronostratigraphiques couvrent les complexes bavéliens, cromériens, l'Holsteinien, le complexe saalien, l'Eemien et le Weichsélien. La mise en contemporanéité avec les stades isotopiques marins indique des faunes présentes du MIS 31 jusqu'à la fin du MIS 2. Cette période correspond intégralement également avec les «Mammal Ages» italiens de la période post-villafranchienne vers 1 million d'années B.P., puis avec le Galérien et 1'Aurélien (Palombo \& Sardella, 2007).

\section{3 - CADRE PALÉOBIOGÉOGRAPHIQUE ET PALÉOÉCOLOGIQUE}

Le cadre paléobiogéographique de la France septentrionale durant le Pléistocène moyen et supérieur est très variable et suit les variations cycliques du contexte climatique. Au cours des phases tempérées, l'Europe du NordOuest constitue une entité cohérente, avec des paramètres sensiblement comparables à l'actuel, un climat apparemment dominé par des influences océaniques, le développement plus ou moins important d'une forêt à feuilles caduques mais où les espaces découverts de type prairie sont néanmoins bien représentés. La Manche et la Mer du Nord sont présentes et isolent les Îles britanniques du continent. Durant les phases froides, la Manche n'existe que sous la forme d'un grand fleuve, permettant le passage des mammifères vers la Grande-Bretagne, la Mer du Nord voit sa limite sud se décaler vers le nord (Lautridou et al., 1999). Le climat est alors nettement continental, sec, le paysage se marque par la présence d'un système de steppes où les zones arborées persistent malgré tout, probablement dans les forêts galeries autour des cours d'eau. Globalement, la France septentrionale se caractérise par des zones aux reliefs peu marqués, formant ainsi la partie occidentale des vastes plaines du nord de l'Europe (Sommé, 1977). Très peu de grottes sont présentes; de fait la totalité des séries fauniques utilisées proviennent de gisements de plein air (fig. 2).

L'évolution des méthodes et surtout des modèles d'analyse paléoécologiques pour le Pléistocène permet maintenant de manière très efficace d'utiliser les grands mammifères en tant que marqueurs environnementaux (Legendre, 1989; Auguste, 1995a; Guérin, 1998) mais également en tant qu'outils chrono-écologiques. La France septentrionale se prête bien à ces analyses et de nombreuses approches pluridisciplinaires développées 


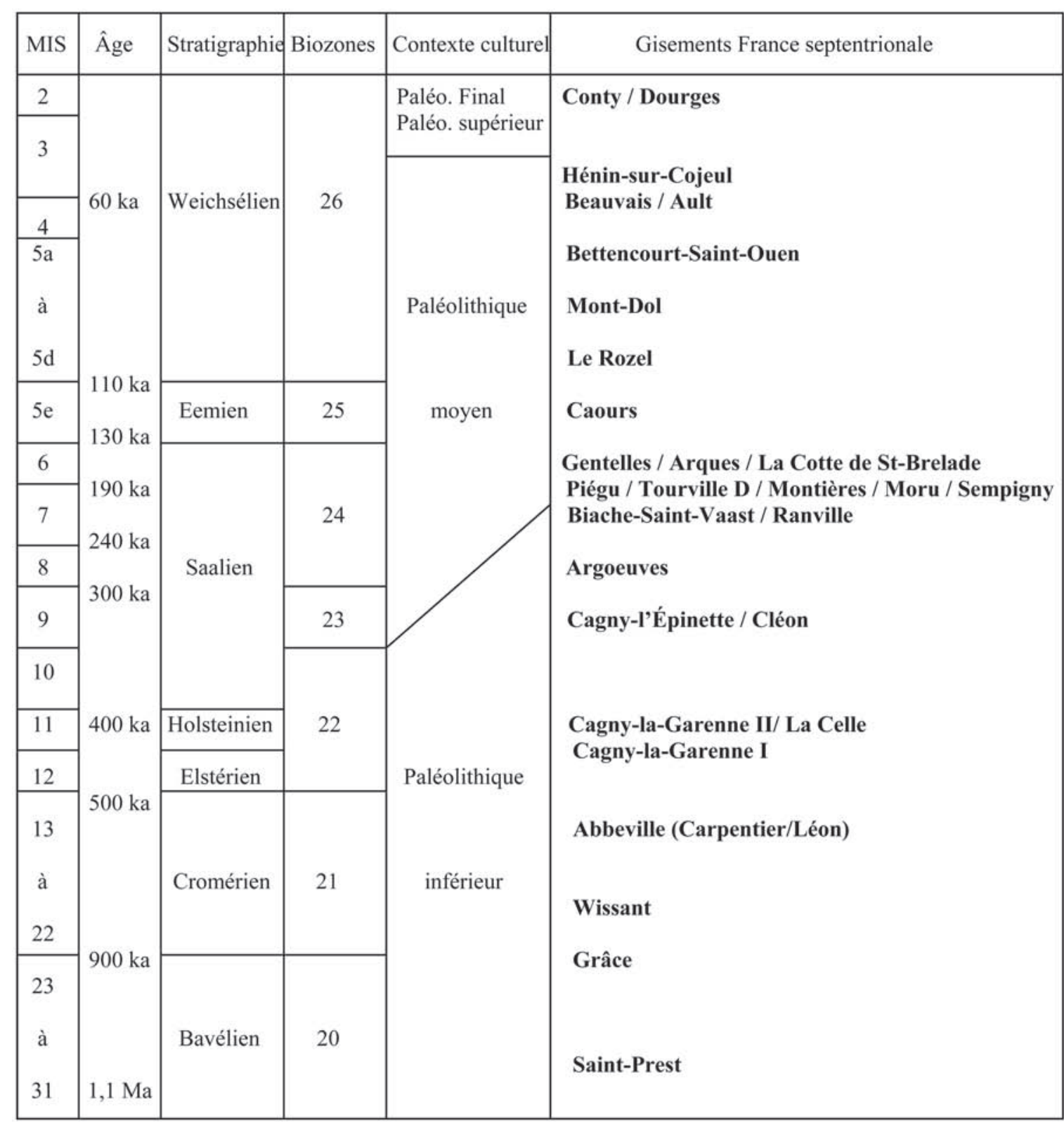

Fig. 1 : Cadres chrono-stratigraphiques, biochronologiques et culturels des gisements à faune mammalienne de France septentrionale (MIS, âges et stratigraphie d'après Gibbard \& Cohen, 2008; biozones d'après Guérin, 1990 ; contexte paléolithique d'après Tuffreau, 1987).

Fig. 1: Chronostratigraphical, biochronological and cultural contexts of Northern France mammals sites (MIS, ages and stratigraphy after Gibbard \& Cohen, 2008; standardzones after Guérin, 1990; paleolithic background after Tuffreau, 1987).

depuis plusieurs années démontrent que les interprétations écologiques et chronologiques induites par les grands mammifères sont en parfait accord avec les résultats des autres disciplines (voir par exemple: Antoine et al., 2003; Antoine et al., 2006; Limondin-Lozouet et al., 2006). Au-delà d'études de séries fauniques provenant de certains gisements en particulier, l'accumulation des données pour le Pléistocène moyen et supérieur nous permet de replacer ces éléments ponctuels au sein d'un schéma global de fonctionnement des écosystèmes pléistocènes. Deux axes majeurs de recherches sont ainsi explorés afin de proposer une modélisation fiable à l'échelle européenne (voire eurasiatique): la prise en compte des mobilités spatiales et temporelles durant cette période; l'utilisation du modèle systémique de la «Steppe à Mammouth» et de son évolution au cours du temps depuis le début du Pléistocène moyen jusqu'à sa disparition à la fin du Pléistocène supérieur.

Les phénomènes de migrations, pris au sens le plus large du terme, font en effet l'objet de recherches depuis de nombreuses années, en particulier par les paléontologues russes (Trofimov, 1955), français (Bonifay, 1969, 1990), allemands (Tobien, 1975), anglais (Lister, 1992; Turner, 1992), néerlandais (Kolfschoten, 1992) et italiens (Palombo, 2007a). Il apparaît clairement dans tous ces travaux que les dynamiques de peuplement d'une région par un groupe ou une association de mammifères sont très complexes. Ceux-ci font intervenir plusieurs facteurs majeurs, tels que l'origine de la spéciation de certaines formes adaptées à des conditions écologiques particulières, les mouvements dans l'espace et le temps («migrations» au sens strict) de ces formes et/ou asso- 


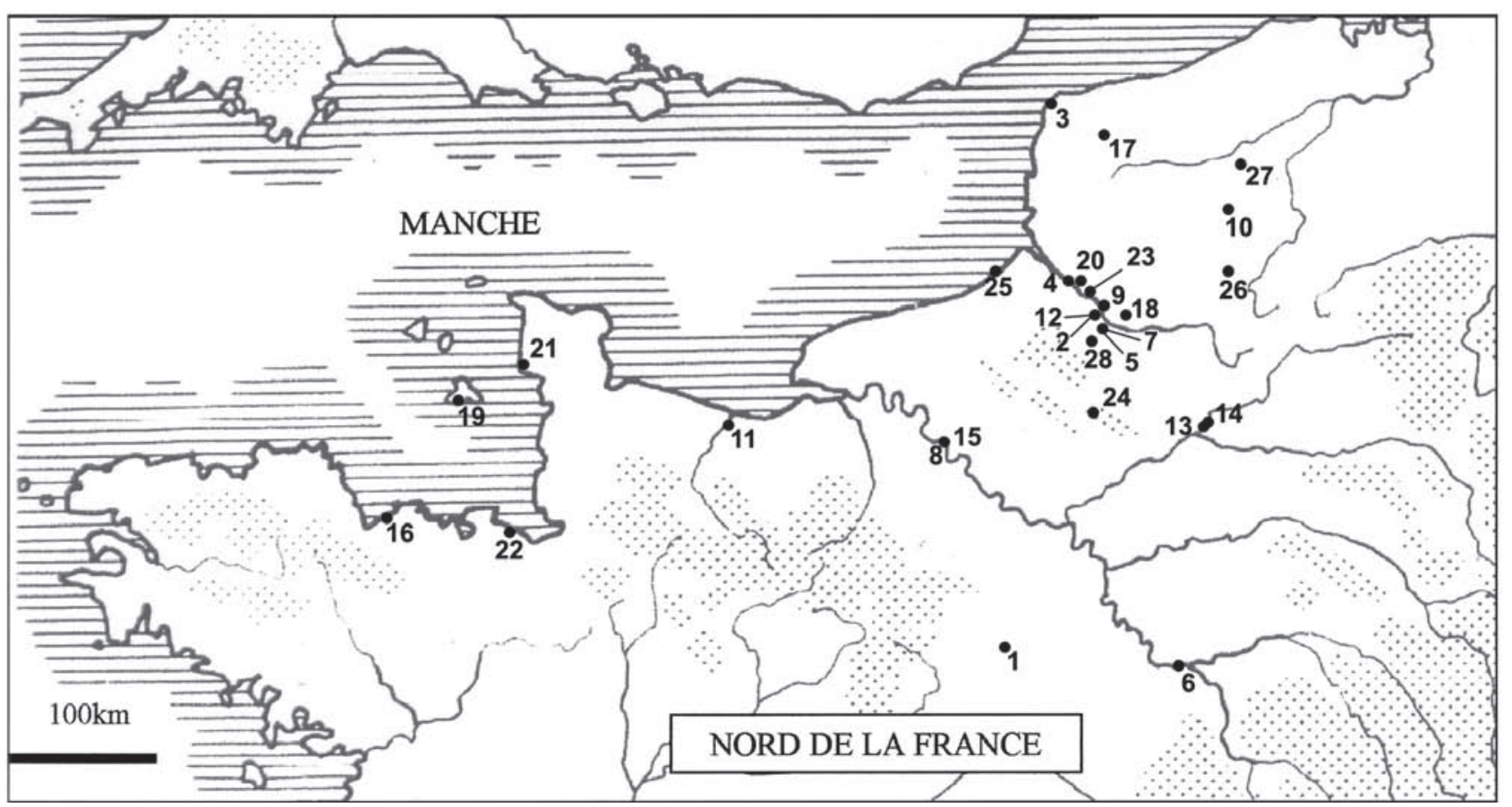

Fig. 2 : Localisation des gisements.

1: Saint-Prest; 2 : Ferme de Grâce; 3: Wissant; 4 : Abbeville, carrières Carpentier et Léon; 5 : Cagny-la-Garenne; $6:$ La Celle ; $7:$ Cagny-l’Épinette; 8 : Cléon; 9: Argœuves; 10: Biache-Saint-Vaast; 11: Ranville; 12: Montières-lès-Amiens, carrière Boutmy-Muchembled; 13: Moru; 14: Sempigny; 15: Tourville-la-Rivière; 16: Piégu; 17: Arques; 18: Gentelles; 19: La Cotte de Saint-Brelade; 20 : Caours; 21 : Le Rozel; 22 : Le Mont-Dol; 23 : Bettencourt-Saint-Ouen; 24 : Beauvais; 25: Ault; 26: Hénin-sur-Cojeul; 27 : Dourges; 28 : Conty.

Fig. 2: Localization of the sites.

ciations fauniques et la colonisation de nouveaux territoires, l'évolution de ces taxons en fonction du temps, la réponse aux variations climatiques puis environnementales à grande échelle géographique. Au sein de chaque zone spatiale, les réponses aux contraintes d'ordre écologique vont de plus différer en fonction de la position biogéographique. C'est ainsi que la couverture végétale de la France septentrionale va répondre de manière plus rapide et plus absolue à des dégradations climatiques, obligeant les herbivores à se déplacer ou à s'adapter s'ils en sont capables aux nouvelles conditions, tandis que dans la zone méditerranéenne, l'atténuation des effets directs des variations du climat permet la persistance de vastes zones refuges pour les végétaux et les animaux adaptés aux contextes tempérés (Bonifay \& Brugal, 1996). De la même manière qu'il existe un gradient de réponse écologique et migratoire global Nord/Sud entre l'Europe du Nord et l'Europe du Sud, il apparaît également qu'un vaste gradient Ouest-Est intervient durant le Pléistocène moyen et supérieur en Eurasie. Celui-ci est généré par l'apparition d'un biome particulier, à l'Est du continent durant le Pléistocène inférieur, où vont évoluer des taxons typiquement adaptés aux conditions climatiques et environnementales des périodes froides qui vont jalonner ensuite le Pléistocène moyen et supérieur. Ce «réservoir» nord-est asiatique de faunes adaptées aux contextes glaciaires va servir de sources cycliques de peuplement durant les phases de péjoration du climat (Kolfschoten, 1992, 1995). L'évolution des mammifères au Pléistocène moyen et supérieur et leur intérêt biochronologique sont donc obligatoirement à replacer dans ce cadre spatio-temporel complexe (Lister, 1997, 2004 ; Palombo, 2007a).
L'écosystème pléistocène dénommé «Steppe à Mammouth» est maintenant assez bien identifié au sein des études paléoécologiques portant sur les mammifères du Pléistocène moyen et supérieur. Ce vaste biome sans équivalent direct actuel est présent durant toute cette période, en gardant intact toutes ses composantes, d'ordre physique tels que les paramètres climatiques, mais aussi d'ordre biologique comme la présence d'une végétation et d'une faune spécifique (Guthrie, 1990). Ce système écologique disparaît à la fin du Pléistocène supérieur pour laisser la place aux biomes holocènes. D'un point de vue faunique, la mise en place des taxons adaptés aux conditions écologiques intrinsèques à ce système s'effectue au cours du Pléistocène inférieur, mais le fonctionnement typique de la «Steppe à Mammouth » n'est effectif qu'à partir de l'extrême fin de cette période et surtout au début du Pléistocène moyen (Kahlke, 1999, 2007). En Europe du Nord-Ouest de manière générale et en France septentrionale en particulier, le schéma migratoire décrit plus haut va fonctionner de manière récurrente, les phases tempérées se caractérisant par la présence de taxons mammaliens adaptés à des paysages dominés par les forêts mais où des zones de prairies sont néanmoins conséquentes, paysages dénommés prairies-parcs boisés (Auguste, 1995a). Dès le début des périodes glaciaires, ces faunes tempérées disparaissent du territoire en migrant vers les zones plus méridionales, pour être remplacées par les formes caractéristiques de la «Steppe à Mammouth » provenant du réservoir nord-est asiatique et sibérien (Kolfschoten, 1992). Le retour à une période tempérée se marque par des flux migratoires des mammifères dirigés en sens contraire au processus précédent. 
Les analyses utilisant les outils et concepts de l'écologie systémique permettent de modéliser et d'interpréter en partie le fonctionnement de cet écosystème pléistocène des périodes froides mais également l'écosystème présent durant les phases tempérées en Europe du Nord-Ouest (Auguste, 1995a). Dans les deux cas, les milieux apparaissent extrêmement favorables à la présence d'une biomasse animale riche et variée, incluant des mammifères de classes de taille allant des très petits animaux (Insectivores) jusqu'aux mégaherbivores (Proboscidiens, Rhinocérotidés et Hippopotamidés) (Guthrie, 1990; Auguste, 1995a; Delpech, 1999). Le rôle des mégaherbivores, mais également des grands herbivores (grands Bovidés, Équidés, grands Cervidés) est essentiel en tant qu'élément régulateur de ces écosystèmes, en maintenant une diversité végétale conséquente permettant une grande variété des sources de biomasse végétale s'opposant nettement à l'uniformisation des milieux homologues holocènes (Owen-Smith, 1988). L'assimilation de la «Steppe à Mammouth» à un système écologique complexe met en évidence les raisons de la stabilité de ses caractères intrinsèques, avec des phénomènes de contrôles positifs et négatifs par les herbivores s'ajoutant aux paramètres de fonctionnement d'autorégulation (Bignon, 2008).

C'est donc au sein de ces écosystèmes récurrents durant le Pléistocène moyen et supérieur que vont s'inscrire les dynamiques des renouvellements des mammifères en France septentrionale.

Les attributions taxinomiques employées dans ce présent travail reposent sur un certain nombre de choix, consensuels ou non. Ainsi, pour les Proboscidiens, les sous-espèces retenues suivent les travaux de Guérin (2007) tout comme pour les Rhinocérotidés (Guérin, 1980 ; 2004), mais les synonymies couramment employées sont mentionnées, le but n'étant pas ici de discuter de cet aspect.

Il est à noter que la disparité des séries fauniques entraîne des différences dans le degré de détermination de plusieurs taxons, certains pouvant suivant les gisements atteindre le niveau sub-spécifique, mais dans la plupart des cas demeurer au stade spécifique.

\section{4 - LES ASSOCIATIONS MAMMALIENNES DU PLÉISTOCÈNE MOYEN ET SUPÉRIEUR EN FRANCE SEPTENTRIONALE}

\section{SAINT-PREST (EURE) (fig. 2, $\mathrm{n}^{\circ} 1$ )}

La plus ancienne faune mammalienne identifiée en France septentrionale est celle de Saint-Prest. Connue depuis le milieu du XIX ${ }^{\mathrm{e}}$ siècle (Boisvillette, 1849), elle a fait l'objet d'une révision globale par Guérin in Guérin et al. (2003). La faune provient de sables fluviatiles d'une ancienne terrasse de l'Eure conservés en contexte karstique. Elle se compose de: Mammuthus meridionalis depereti, Dicerorhinus etruscus brachycephalus (=Stephanorhinus hundsheimensis), Equus stenonis, Alces carnutorum, Megaloceros verticornis
(= Praemegaceros verticornis), Cervus $\mathrm{cf}$. elaphus, aff. Dama sp., Bison schoetensacki, Hippopotamus major, Trogontherium cuvieri boisvilletti et Pachycrocuta brevirostris. Cette association mammalienne est typique de la biozone 20, vers 1 / 0,9 million d'années BP. Elle caractérise un milieu à dominante forestière avec présence d'espaces découverts, sous un climat tempéré humide. Elle s'apparente en partie avec plusieurs faunes de gisements européens comme Sainzelles (Haute-Loire), Durfort (Gard), Le Vallonnet (Alpes-Maritimes), Rosières (Cher), Les Valerots (Côte-d'Or) et Untermassfeld (Allemagne) (Guérin, 1980; Moullé, 1997-1998; Kahlke, 2007). Cette période apparaît fondamentale en tant qu'événement de renouvellement majeur des peuplements mammaliens en Europe, avec la présence de nouveaux taxons ou stades évolutifs pour certaines espèces, phénomène migratoire et évolutif contemporain d'une période tempérée du Bavélien. Les associations mammaliennes de cette période représentent une phase de rupture entre les faunes villafranchiennes du Pléistocène inférieur et les faunes «typiques» quaternaires du Pléistocène moyen et supérieur (Kahlke, 2007).

\section{FERME DE GRÂCE (SOMME) (fig. 2, n 2)}

Le gisement de la Ferme de Grâce à Montières, près d'Amiens, a livré quelques artefacts et une seule dent d'Équidé au sommet d'un niveau de cailloutis, correspondant à la fin d'une phase froide, constituant un des témoins de la très haute terrasse de la Somme (Bourdier, 1969; Bourdier et al., 1974a). La polarité magnétique de cette séquence est négative, donc antérieure à la limite Matuyama-Brunhes (Biquand, 1974). L'âge du niveau concerné n'est pas établi avec certitude, mais se place vers la fin du Bavélien ou le début du Cromérien (MIS 23/22) vers 900000 ans BP (Sommé, 1989; Antoine et $a l .$, 2007). La dent d'Équidé, étudiée par F. Prat, a été rapportée à Equus stenonis, mais pourrait appartenir à Equus altidens.

\section{WISSANT (PAS-DE-CALAIS) (fig. 2, $\mathrm{n}^{\circ} 3$ )}

La baie de Wissant présente des séquences sédimentaires pléistocènes dont la stratigraphie a pu être précisée (de Heinzelin, 1964). Un cailloutis de base a livré quelques vestiges fauniques qui permettent, en accord avec le cadre géomorphologique, de situer chronologiquement le niveau fossilifère (Bouchud, 1964, 1968; Poplin, 1969; Sommé \& Tuffreau, 1976). Deux espèces ont été identifiées: Mammuthus meridionalis cromerensis et Hippopotamus major. Le mammouth est plus évolué que celui de Saint-Prest et correspond à la dernière sous-espèce connue pour ce taxon (Bouchud, 1968). L'hippopotame correspond bien à la grande forme $H$. major, déterminée par Poplin (1969) et confirmé par la suite par Faure (1983). Cette espèce, disparaissant à la fin de la biozone 21, associée avec la forme évolué du mammouth méridional, indique un âge cromérien ancien vers $800000 / 700000$ ans BP (MIS 21/17), durant une phase tempérée. 


\section{ABBEVILLE, CARRIERES CARPENTIER ET LÉON (SOMME) (fig. 2, n 4)}

Parmi les nombreuses carrières exploitées dès le $\mathrm{XIX}^{\mathrm{e}}$ siècle à Abbeville, c'est essentiellement celle de la Carrière Carpentier qui présente un intérêt majeur de par la découverte d'artefacts lithiques et surtout de vestiges fauniques de mammifères. Étudiée en détail par Commont (1910), ce gisement présente une stratigraphie incluse pro-parte dans la haute terrasse de la Somme, où un niveau en particulier livre l'essentiel de la faune classiquement citée dans la littérature, celui dit de la «marne blanche» (Commont, 1910; Tuffreau, 1987). Les vestiges mammaliens qui y ont été découverts ont fait l'objet de très nombreuses études depuis la fin du XIX ${ }^{\mathrm{e}}$ siècle (Gaudry in d'Ault du Mesnil, 1896; Boule in Commont, 1910 ; Pontier, 1910, 1928; Prat, 1968a, 1980; Guérin, 1980; Faure, 1983, 1984, 1985 ; Auguste, 1995a, 1995b). C'est de la partie inférieure de cette marne blanche que provient la série faunique la plus caractéristique (Auguste, 1995b; Auguste in Schreve et al., 2007), qui se compose des taxons suivants: Мammuthus meridionalis, Palaeoloxodon antiquus, Dicerorhinus etruscus brachycephalus (= Stephanorhinus hundsheimensis), Hippopotamus incognitus, Sus scrofa mosbachensis, Equus de type sténonien, Equus mosbachensis, Homotherium latidens, Megaloceros verticornis (= Praemegaceros verticornis), Bison sp. et Bos primigenius. Cette faune témoigne de conditions écologiques caractérisées par un paysage de forêt et d'espaces de prairie, des zones marécageuses, sous un climat nettement tempéré et humide. Ces interprétations concordent assez bien avec celles fournies par les analyses palynologiques réalisées dans la marne blanche qui indiquent un milieu de sylvo-steppe sous un climat tempéré continental (Munaut, 1989). Ce cortège mammalien s'apparente en partie avec les faunes classiques décrites dans le Cromer Forest Bed anglais à West Runton (Stuart, 1975, 1981), mais en diffère par des taxons témoignant de conditions climatiques plus clémentes. Toutefois, d'un point de vue évolutif, les taxons sont identiques. Une analogie plus nette existe avec la faune typiquement tempérée de Pakefield en Angleterre datée du MIS 17 ou 15 (Stuart \& Lister, 2001), ou celle du niveau principal «graues» de Mosbach en Allemagne (Brüning, 1980). L'association faunique de la marne blanche de la carrière Carpentier à Abbeville est donc typique du Cromérien en contemporanéité avec une phase nettement tempérée très probablement corrélable avec le MIS 15 et caractérise bien la biozone 21. Ces interprétations sont en parfait accord avec le cadre géomorphologique (Antoine et al., 2007 ) et les datations radiométriques (ESR sur quartz) qui donnent un âge moyen de $600000 \pm 90000$ ans BP (Laurent, 1993).

La carrière Léon, située dans le même contexte stratigraphique, a livré quelques artefacts et des vestiges de faune mammalienne comparables à ceux de la marne blanche de la carrière Carpentier.

\section{CAGNY-LA-GARENNE (SOMME) (fig. 2, n 5)}

La carrière classique de Cagny-la-Garenne est connue pour les très nombreuses industries acheuléennes qui y ont été découvertes au sein de nappes alluviales de l'Avre, affluent de la Somme, correspondant au système de la moyenne terrasse de la Somme. Sa stratigraphie complexe a pu être établie (Bourdier, 1969; Bourdier et al., 1974b; Haesaerts \& Dupuis, 1986; Antoine, 1990). La position des artefacts et de la faune a été précisée par des fouilles menées durant les années 1980 par A. Tuffreau (Tuffreau, 1987). La faune, qui provient de la base de la séquence au sommet d'un cailloutis, est représentée par (Prat, 1968a; Bourdier et al., 1974b; Auguste, 1995a, 1995b): grand Bovidé, Mammuthus trogontheri, Equus mosbachensis, Cervus sp. Cette série faunique indiquerait plutôt un paysage découvert de type steppique dans un contexte climatique plutôt froid. Sa position en dessous d'une séquence sédimentaire formée en contexte tempéré pourrait indiquer une faune tardiglaciaire incluse au sein de la biozone 22, probablement vers la fin du MIS 12, interprétation compatible avec les données géomorphologiques (Antoine, 1990) et des datations par ESR sur quartz donnant un âge moyen de $400000 \pm 101000$ ans BP (Laurent, 1993).

Des fouilles menées durant les années 1990-2000 dans un autre secteur de Cagny-la-Garenne, nommé Garenne II, ont permis de mettre au jour de nouveaux vestiges fauniques en association avec une industrie acheuléenne (Tuffreau, 2001). La faune, qui provient des niveaux I et $\mathrm{H}$, se différencie de celle de la carrière classique par une caractérisation d'un climat tempéré dans un environnement de type prairie parc-boisé (Auguste in Schreve et al., 2007): Bos primigenius, Equus sp. type Hoxne, Canis lupus mosbachensis et Cervus cf. elaphus. La présence du premier vrai loup sous sa forme primitive constitue un bon marqueur biochronologique, information complétée par un cheval de type caballin comparable aux formes anglaises identifiées à Hoxne, Clacton et Swanscombe (Eisenmann, 1991). La faune s'inscrit bien au sein de la biozone 22. Ces gisements sont attribués à une phase très tempérée contemporaine du MIS 11 et de 1'Holsteinien (dénommé Hoxnien en Angleterre). La mise en corrélation de la faune de la Garenne II avec l'interglaciaire holsteinien et le MIS 11 s'accorde bien avec la position au sein de la terrasse et les âges radiométriques obtenus de l'ordre de $448000 \pm 68000$ ans BP (Antoine et al., 2007; Bahain et al., 2007).

\section{LA CELLE (SEINE-ET MARNE) (fig. $2, n^{\circ}$ 6)}

Le gisement de La Celle-sous-Moret (maintenant nommé La Celle-sur-Seine) est connu depuis la fin du $\mathrm{XIX}^{\mathrm{e}}$ siècle par des découvertes paléontologiques et quelques industries humaines, en particulier des empreintes foliaires conservées au sein d'un sédiment tufacé. Des campagnes récentes de travaux pluridisciplinaires ont permis d'une part de préciser le contexte stratigraphique et chronologique, et d'autre part de retrouver des vestiges de faune mammalienne et quelques artefacts 
dans le cadre du programme «SITEP» du CNRS (Eclipse) sous la direction de N. Limondin-Lozouet (Limondin-Lozouet et al., 2006). La faune se situe au sein d'un niveau limoneux inséré dans une grande série sédimentaire de tufs, en position de moyenne terrasse de la Seine. Elle comprend les taxons suivants (Auguste in Limondin-Lozouet et al., 2006): Cervus elaphus, Equus sp. type Hoxne, Macaca sylvanus, Hippopotamus incognitus et un petit Carnivore non identifié. La faune traduit une période nettement tempérée et humide et un paysage dominé par la forêt. Le cheval s'apparente également aux chevaux anglais contemporains de l'Holsteinien et corrélés avec le MIS 11, parfaitement en accord avec la position stratigraphique, la caractérisation du cortège malacologique («faune à Lyrodiscus») témoignant de l'optimum climatique du MIS 11, ainsi que les datations radiométriques par ESR et U-Th sur dent de cheval donnant un âge moyen de $424000 \pm 38000$ ans BP (Limondin-Lozouet et al., 2006; Antoine et al., 2007; Bahain et al., sous presse). La présence du macaque est la première mention (et la seule actuellement) en France septentrionale. L'hippopotame est signalé pour la première fois en Europe pour l'interglaciaire contemporain du MIS 11 (Auguste in Schreve et $a l .$, 2007). Cette faune apparaît très comparable avec celle de la Garenne II.

\section{CAGNY-L'ÉPINETTE (SOMME) (fig. 2, n 7)}

Le gisement de Cagny-l'Épinette, situé non loin de celui de la Garenne, est fouillé par A. Tuffreau depuis le début des années 1980. Plusieurs niveaux archéologiques contenant de l'industrie acheuléenne et de la grande faune mammalienne ont été identifiés au sein d'un cadre stratigraphique permettant d'attribuer le dépôt à la moyenne terrasse de la Somme, postérieurement à celle de la Garenne (Tuffreau et al., 1995). Le cadre écologique des dépôts fossilifères et paléolithiques des niveaux fluviatiles est bien connu grâce à plusieurs études concernant la grande et microfaune mammalienne, les mollusques et les pollens. Le climat est tempéré continental et le paysage constitué par une mosaïque de forêt boréale et de steppe (Tuffreau et al., 1995; Lamotte et al., 2005). L'âge des dépôts fluviatiles a pu être estimé à une période tempérée intra-saalienne contemporaine du MIS 9 avec des dates obtenues par ESR sur quartz de $296000 \pm 53000$ ans BP, ESR et U-Th sur dents d'aurochs de $318000 \pm 48000,289000 \pm$ 43000 et $291000 \pm 44000$ ans BP (Laurent, 1993; Bahain et al., 2007). La grande faune a fait l'objet pour la première phase des fouilles d'une étude par A.-M. Moigne et a livré le cortège suivant (Moigne, 1988, 1989): Crocuta crocuta spelaea, Bos primigenius, Cervus elaphus, Dama dama, Equus mosbachensis et cf. Palaeoloxodon antiquus. Depuis 1997, nous avons repris l'étude du matériel issu des fouilles menées tous les ans, mais nous n'avons retrouvé que trois taxons déjà identifiés (Auguste in Lamotte et al., 2005): Bos primigenius, Cervus elaphus et Equus cf. mosbachensis. Cette faune tempérée s'inscrit au sein de la biozone 23 et s'accorde bien avec l'âge proposé pour la séquence.

\section{CLÉON (SEINE-MARITIME) (fig. $2, n^{\circ} 8$ )}

Le gisement de Cléon, inclus dans un contexte de terrasse de la Seine, a livré quelques vestiges fauniques de grands mammifères au sein d'une séquence stratigraphique complexe. L'étude du matériel a permis de clarifier l'incertitude sur l'âge de cette faune et sa corrélation avec celle du gisement de Tourville-la-Rivère dont les dépôts sont très proches (Auguste et al., 2003). La faune provient uniquement de sédiments fluviatiles et comprend du matériel en place et en position secondaire. Elle se compose de: Cervus elaphus, Bos primigenius, Dicerorhinus hemitoechus (= Stephanorhinus hemitoechus), Equus sp. et Palaeoloxodon antiquus. C'est la première apparition en France septentrionale du rhinocéros de prairie. Cet assemblage témoigne de conditions climatiques clémentes et d'un milieu de forêts et de prairies. Il a été mis en corrélation avec la partie inférieure de la séquence de Tourville, durant un épisode intrasaalien tempéré contemporain du MIS 9.

\section{ARGEEUVES (SOMME) (fig. 2, $\mathrm{n}^{\circ}$ 9)}

Le gisement d'Argœuves, près d'Amiens, a livré au sein de graviers périglaciaires de la basse terrasse de la Somme des industries moustériennes et quelques vestiges de grande faune (Bourdier, 1969; Bourdier et al., 1974b). Seules deux espèces ont été identifiées (Prat in Bourdier et al., 1974b; Guérin, 1980): Coelodonta antiquitatis et Equus $\mathrm{cf}$. steinheimensis. Ces deux taxons témoignent bien de conditions écologiques cohérentes avec leur position au sein de graviers déposés en période froide, un climat froid, sec et continental et un paysage découvert de type steppique. C'est la première apparition du rhinocéros laineux en France septentrionale. L'âge saalien de cette faune est assuré par sa situation en contexte de basse terrasse, donc postérieure au premier interglaciaire intra-saalien identifié à Cagny-l'Épinette, et par cette première apparition du rhinocéros laineux, en contemporanéité avec une phase froide corrélable avec le MIS 8, caractérisant le début de la biozone 24 .

\section{BIACHE-SAINT-VAAST (PAS-DE-CALAIS) (fig. 2, $\mathrm{n}^{\circ} 10$ )}

Le gisement de Biache-Saint-Vaast a livré une très riche accumulation faunique associée avec une industrie moustérienne au sein d'une séquence fluviatile, surmontée par des dépôts de versant puis des lœss (Tuffreau \& Sommé, 1988). Deux crânes humains présentant les caractères diagnostiques des Néandertaliens ont également été découverts au sein du niveau fluviatile IIa, faisant de ce gisement le plus ancien de France septentrionale à avoir livré des éléments paléoanthropologiques (Vandermeersch, 1978, 1982; Rougier, 2003; Guipert, 2005). La séquence s'inscrit en position de basse terrasse de la Scarpe, en contemporanéité avec le second épisode interglaciaire intra-saalien corrélé avec le MIS 7, au moins pour les dépôts fluviatiles (Tuffreau 
\& Sommé, 1988). Des datations utilisant plusieurs méthodes et supports indiquent un âge global compris entre $263000+53000 /-37000$ ans BP et 175000 ans $\pm 13000 \mathrm{BP}$ pour le niveau IIa, mais de nouvelles études ont donné pour ce même niveau un âge par ESR sur dents et ossements de $229000 \pm 27000$ et 230000 \pm 24000 ans BP (Bahain et al., 1993; Bahain, 2007). La faune comprend plus de 220000 vestiges fauniques dont plus de 20000 ont pu être déterminés anatomiquement et spécifiquement (Auguste, 1995a). La séquence fluviatile comprend 20 espèces de grands et méso-mammifères : Canis lupus cf. mediterraneus, Vulpes vulpes, Felis silvestris, Panthera spelaea, Ursus arctos, Ursus deningeri cf. hercynicus, Aonyx antiqua, cf. Martes martes, Sus scrofa scrofa, Cervus elaphus elaphus, Megaloceros giganteus, Capreolus capreolus capreolus, Bos primigenius, Equus cf. taubachensis, Equus hydruntinus, Dicerorhinus hemitoechus (= Stephanorhinus hemitoechus), Dicerorhinus mercki (= Stephanorhinus kirchbergensis), Palaeoloxodon antiquus et Castor fiber. Cette faune très homogène indique une prairie parc-boisé sous un climat tempéré et humide, en accord avec les autres reconstitutions paléoécologiques (Auguste, 1995a). Le degré évolutif atteint par certains taxons, la position stratigraphique de cette faune, ses composantes écologiques et sa contemporanéité avec un optimum climatique plaide pour une phase ancienne au sein du MIS 7, vers 230000 ans BP. Elle est typique de la biozone 24 .

Les deux derniers niveaux archéologiques et fossilifères de Biache, les niveaux D et D1 formé en contexte de début de dépôt de lœss, ont livré une faune moins riche et sensiblement différente (Auguste, 1995a): Vulpes vulpes, Cervus elaphus, Bos primigenius, Equus cf. mosbachensis, Equus hydruntinus, Coelodonta antiquitatis praecursor et Palaeoloxodon antiquus. Cette faune présente une mosaïque de taxon indiquant un climat plus froid, plus sec et plus continental que dans les niveaux sous-jacents, dans un milieu également beaucoup plus ouvert où la steppe fait son apparition. L'attribution chronologique de cette faune est celle de la fin du stade isotopique 7 .

\section{RANVILLE (CALVADOS) (fig. 2, $\mathrm{n}^{\circ} 11$ )}

Découvert en 2000, le gisement de Ranville, situé près de Caen, constitue un cas très exceptionnel de conservation en contexte karstique d'un niveau paléolithique en position secondaire (Cliquet, 2008). Formé initialement durant une phase tempérée de la fin du Pléistocène moyen en contexte fluviatile, le site a connu un épisode de sous-tirage karstique qui a entraîné une grande partie du sédiment et des vestiges lithiques et fauniques qui s'y étaient déposés au sein de deux puits. L'industrie s'inscrit au sein de la variabilité observée à la fin du Pléistocène moyen pour les assemblages du Paléolithique moyen ancien. Des datations par U-Th et RPE sur des dents d'herbivores ont donné un âge moyen d'environ 216000 \pm 9000 ans BP, soit une occupation humaine durant un épisode interglaciaire intra-saalien contemporain du MIS 7 (Bahain et al., 2008). La faune se compose des espèces suivantes (Auguste, 2008): Palaeoloxodon antiquus, Dicerorhinus mercki (= Stephanorhinus kirchbergensis), Bos primigenius, Equus sp., Equus hydruntinus, Cervus elaphus, Canis lupus et Vulpes vulpes. L'association mammalienne témoigne d'un climat tempéré et d'un paysage en mosaïque de forêts et de prairies, corrélable avec le second épisode interglaciaire intra-saalien et le MIS 7.

MONTIÈRES-LES-AMIENS (SOMME) (fig. 2, n 12)

Parmi les nombreuses carrières situées sur la zone de Montières, près d'Amiens, celle de Boutmy-Muchembled est la plus connue et la plus intéressante. En position de basse terrasse de la Somme, cette carrière a été étudiée en détail par Commont qui y avait décrit une «faune chaude» associée avec une industrie qu'il avait dénommée "Moustérien à faune chaude» au sein de niveaux sableux sus-jacents aux graviers de base (Commont, 1912). Le cadre écologique avait bien été mis en évidence par les études malacologiques de Wüst (cité in Commont, 1912), avec un climat tempéré et un couvert forestier notable. La faune mammalienne comprend (Prat, 1968a ; Guérin, 1980 ; Faure, 1983 ; Auguste, 1995a, 1995b): Hippopotamus incognitus, Palaeoloxodon antiquus, Dicerorhinus mercki (= Stephanorhinus kirchbergensis), Equus hydruntinus, Equus sp. de type caballin, Panthera spelaea, Cervus elaphus, Megaloceros giganteus, Bos primigenius et Ursus arctos. Ce cortège mammalien témoigne également d'un climat nettement tempéré et d'un milieu de forêts, d'espaces découverts herbeux et de zones marécageuses. Elle s'inscrit au sein de la biozone 24. D'après sa position, la basse terrasse de Montières est postérieure à celle d'Argœuves et est donc attribuable à la fin de l'interglaciaire intrasaalien, en contemporanéité avec le MIS 7 et plus précisément le second épisode tempéré corrélé avec le MIS 7a vers 200000 ans BP (Haesaerts \& Dupuis, 1986; Antoine, 1990). La datation de ces niveaux fossilifères et archéologiques par ESR sur quartz confirme cette attribution chronologique avec un âge de 200000 \pm 57000 ans BP (Laurent, 1993).

\section{MORU (OISE) (fig. 2, $\mathrm{n}^{\circ} 13$ )}

La basse terrasse de l'Oise à Moru a livré des artefacts paléolithiques associés avec des vestiges fauniques au sein de sédiments sableux. Initialement attribuée au dernier interglaciaire (Patte, 1924, 1941; Prat, 1968b; Bourdier, 1969), la séquence stratigraphique appartient en fait comme les autres dépôts similaires du Nord de la France à la dernière phase tempérée intra-saalienne (Tuffreau et al., 1981). La faune est composée des espèces suivantes (Patte, 1924 ; Prat, 1968b ; Guérin, 1980 ; Faure, 1983, Auguste, 1995a): Crocuta crocuta spelaea, Palaeoloxodon antiquus, Hippopotamus incognitus, Dicerorhinus mercki (= Stephanorhinus kirchbergensis), Equus cf. steinheimensis, Bos primigenius, Cervus elaphus, Megaloceros giganteus, Coelodonta antiquitatis et Mammuthus trogontheri-primigenius (= Mammuthus intermedius ? ou Mammuthus trogontheri 
évolué?). Un mélange de faune apparaît avec la présence du rhinocéros laineux et du mammouth, ceux-ci provenant probablement de dépôts plus anciens remaniés lors de la formation du niveau sableux (Patte, 1941). Si l'on exclut ces deux taxons, la faune apparaît très homogène et caractérise bien un climat nettement tempéré humide et un paysage diversifié de zones forestières, herbeuses et marécageuses. Cette faune s'apparente avec celle de Montières et dans une moindre mesure celle de Biache. Elle caractérise la biozone 24, et correspond bien avec la dernière phase interglaciaire du Pléistocène moyen aux environs de 200000 ans BP et le MIS 7 (7a?).

\section{SEMPIGNY (OISE) (fig. $\left.2, \mathrm{n}^{\circ} 14\right)$}

En amont de Moru, dans la même position de basse terrasse de l'Oise, un niveau de sédiments fluviatiles a livré une industrie très comparable à celle de Moru (Paléolithique moyen ancien) accompagnée par des vestiges mammaliens (Patte, 1967). Cette faune comprend (Patte, 1967; Guérin, 1980; Auguste, 1995a): Mammuthus primigenius, Coelodonta antiquitatis, Equus sp. de type caballin, Equus hydruntinus, Sus scrofa, Cervus elaphus, Megaloceros giganteus, Panthera spelaea, Crocuta crocuta spelaea, Dicerorhinus mercki (= Stephanorhinus kirchbergensis) et Dicerorhinus etruscus brachycephalus (= Stephanorhinus hundsheimensis). La mention de la hyène n'est pas liée à sa présence parmi les restes osseux, mais est induite par des marques typiques de rognage visibles sur un os de rhinocéros de Merck, stigmates attribués initialement à une action anthropique (Patte, 1967, fig. 6). Cette accumulation apparaît assez hétérogène et trois groupes sont discernables: le mammouth et le rhinocéros laineux, tout comme à Moru, proviennent très probablement du remaniement d'un dépôt plus ancien formé durant une phase froide; la présence du rhinocéros étrusque est anachronique et témoigne d'un transport de l'amont depuis un dépôt bien plus ancien pouvant être d'âge bavélien ou cromérien comme le suggère Guérin (1980). Une fois ces trois espèces écartées, les autres taxons forment une association indiquant un environnement à la fois boisé et découvert comprenant des zones de prairie et de marécages, sous un climat tempéré humide. Une mise en contemporanéité de cette faune avec le dernier épisode tempéré intra-saalien et la fin du MIS 7 est tout à fait cohérente.

\section{TOURVILLE-LA-RIVIÈRE}

(fig. $2, \mathrm{n}^{\circ} 15$ )

\section{(SEINE-MARITIME)}

Le gisement de Tourville-la-Rivière se situe en contexte de basse terrasse de la Seine, non loin du gisement de Cléon. La stratigraphie montre la succession de deux cycles glaciaires comportant chacun une phase tempérée bien individualisée dont les datations par thermoluminescence sur sédiment sont respectivement de $314000 \pm 32000$ ans BP et de $196000 \pm 23000$ ans BP, et par ESR sur coquille pour le deuxième interglaciaire de 200000 ans BP (Stremme, 1985; Balescu et al., 1997; Lautridou et al., 1999; Lautridou, 2003). Une très riche collection de grands mammifères a été découverte au sein de cinq niveaux, dont une partie a fait l'objet d'une étude détaillée et systématique (Descombes, 1980, 1982, 1983 ; Beden \& Descombes, 1982; Descombes \& Carpentier, 1987). Parmi ces séries, c'est celle du niveau D1, correspondant au deuxième interglaciaire de Tourville, qui est la plus importante et présente le plus de taxons: Canis lupus, Vulpes vulpes, Crocuta crocuta spelaea, Meles meles, Martes sp., Lutra sp., Ursus arctos, Ursus cf. spelaeus, Capreolus capreolus, Cervus elaphus, Megaloceros giganteus, Bison priscus, Bos primigenius, Sus scrofa, Coelodonta antiquitatis, Equus cf. mosbachensis, Lepus sp. Cette faune témoigne bien d'un climat tempéré mais continental dans un milieu en mosaïque de sylvosteppes et de zones marécageuses. Elle s'intègre bien au sein de la biozone 24. Cette série faunique du niveau D1 de Tourville s'apparente assez bien avec son homologue à Biache, pour les niveaux D et D1, et est donc corrélable avec la fin du dernier interglaciaire intra-saalien et la fin du MIS 7 (MIS 7a), en parfait accord avec les datations de ce niveau qui le situe vers 200000 ans BP.

\section{PIÉGU (CÔTES-D’ARMOR) (fig. 2, n 16)}

Le gisement de Piégu fait partie d'un ensemble de stations paléolithiques du littoral de la région de Pléneuf-Val-André, ayant parfois livré quelques pièces fauniques (Monnier, 1991). La stratigraphie du gisement montre une succession complexe de plusieurs niveaux représentant au moins quatre cycles glaciaires. C'est au sein du niveau $\mathrm{G}$, qui correspond à des éboulis de blocaille consécutif à l'érosion d'un site paléolithique situé au sommet de la falaise de Piégu, que la faune a été retrouvée (Hallegouët et al., 1993). Des datations par ESR et par U-Th sur dents et ossements de cerf donnent des âges de $173000 \pm 13000$ et 198000 \pm 17000 ans BP (Bahain, 2007). Encore inédite en tant que série décrite, la faune a fait l'objet de premiers travaux par J.-C. Descombes, puis F. Serre. Une étude détaillée du cerf élaphe, espèce très largement dominante à Piégu, a été réalisée par Liouville (2007). Nous avons parallèlement repris l'intégralité du matériel qui livre les taxons suivants (Auguste et al., 2005): Canis lupus, Dicerorhinus cf. hemitoechus, Equus sp. de grande taille, Cervus elaphus, Capreolus capreolus, Bos primigenius, Sus scrofa, Megaloceros giganteus et un Lagomorphe (cf. Oryctolagus sp.). Cette association faunique témoigne d'un climat tempéré humide et d'un paysage dominé par la forêt avec présence d'espaces découverts herbeux. Elle s'inscrit bien au sein de la biozone 24. Ces deux paramètres et l'analogie de cette série mammalienne de Piégu avec les séries du dernier interglaciaire intra-saalien précédemment décrites plaideraient pour une contemporanéité avec le MIS 7. Les datations montrent que cette faune peut être corrélée avec la fin du MIS 7. 


\section{ARQUES (PAS-DE-CALAIS) (fig. 2, $\mathrm{n}^{\circ}$ 17)}

La vallée de l'Aa dans le Nord de la France présente un système de terrasse identique à celui de la vallée de la Somme, avec trois terrasses identifiées. Le complexe de la basse terrasse est le seul à avoir livré des fossiles (Sommé, 1977; Tuffreau, 1987). Les travaux de G. Pontier sur cette basse terrasse, menés de la fin du $\mathrm{XIX}^{\mathrm{e}}$ siècle jusqu'au début du $\mathrm{XX}^{\mathrm{e}}$ siècle, ont permis en particulier la découverte de fossiles dans la carrière de la Garenne à Arques, où une industrie moustérienne est associée avec de la faune au sein de deux cailloutis (Pontier, 1914). La faune du cailloutis inférieur comprend (Pontier, 1914; Auguste, 1995a): Mammuthus trogontheri, Coelodonta antiquitatis, Equus sp., Bos primigenius, Capreolus capreolus, Cervus elaphus et Bison priscus. Les espèces provenant du cailloutis supérieur sont: Mammuthus primigenius, Coelodonta antiquitatis, Equus sp., cf. Bos primigenius, Rangifer tarandus et Cervus elaphus. Le mammouth est représenté de manière exceptionnelle par un squelette subcomplet. L'étude de sa denture confirme bien son appartenance à une forme déjà assez typique de l'espèce. La faune du cailloutis inférieur indique un paysage steppique mais où persistent des zones boisées sous un climat continental et plutôt froid. Pour le cailloutis supérieur, le milieu est plus nettement steppique et le climat également plus froid et nettement continentalisé et sec. Chronologiquement, la faune s'inscrit au sein de la biozone 24, et semble témoigner de deux phases temporellement très éloignées l'une de l'autre, la plus ancienne durant une période froide qui pourrait être contemporaine du MIS 8, la seconde contemporaine du MIS 6. Cette interprétation est en accord avec les données géomorphologiques (Sommé, 1977).

\section{GENTELLES (SOMME) (fig. 2, $\mathrm{n}^{\circ} 18$ )}

Le gisement du Mont de l'Évangile à Gentelles occupe une position très peu courante pour les gisements paléolithiques du Nord de la France, il correspond en effet à l'occupation d'un système de dolines développées au sommet d'un plateau crayeux, à l'interfluve entre la Somme et l'Avre. La stratigraphie du gisement est complexe, mais au moins quatre cycles sédimentaires correspondant à quatre cycles glaciaires ont été identifiés dans lesquels une abondante industrie acheuléenne à bifaces a été découverte (Tuffreau et al., 2001). Seule l'unité stratigraphique du cycle III a livré quelques vestiges fauniques. Cette unité correspond à une séquence limoneuse à caractère lœssique surmonté par un sol brun lessivé interglaciaire. Des datations de l'unité «Lag» par IRSL sur sédiment donnent un âge de 194000 \pm 21000 ans BP, soit le début du MIS 6 (Balescu \& Tuffreau, 2004), des datations ESR/U-series sur dents de cheval du niveau archéologique LGC inclus dans cette unité sont de $199000 \pm 20000$ ans BP (Bahain et al., sous-presse). L'essentiel du matériel faunique provient du niveau LGC et est encore inédit (Auguste, sous presse b). À l'exception d'un seul fragment dentaire de grand Bovidé provenant d'un niveau plus ancien, tous les vestiges découverts appartiennent à Equus cf. mosbachensis. Ce cheval présente des affinités avec la forme décrite à Tourville-la-Rivière pour le niveau D1, et diffère sensiblement du Cheval des niveaux fluviatiles de Biache. Il pourrait témoigner d'un contexte plutôt découvert sous un climat non tempéré mais pas forcément glaciaire. La position biochronologique et l'adaptation écologique de ce cheval sont donc en accord avec sa présence au sein d'un dépôt lœssique corrélé avec le tout début du MIS 6.

LA COTTE DE SAINT-BRELADE (JERSEY) (fig. 2, $\left.n^{\circ} 19\right)$

Le gisement de la Cotte de Saint-Brelade se situe dans l'île anglo-normande de Jersey qui, durant les phases glaciaires, étaient régulièrement reliée au continent. Connu depuis le début du $\mathrm{XX}^{\mathrm{e}}$ siècle et fouillé durant les années 1960-1970, ce gisement a permis la découverte de plusieurs accumulations de grands mammifères associées avec une industrie du Paléolithique moyen (Callow \& Cornford, 1986). Deux complexes sédimentaires ont été mis en évidence, le plus ancien comprenant les ensembles II et III d'où provient l'essentiel du matériel lithique et faunique. Le second complexe comprend l'ensemble $\mathrm{V}$ avec des artefacts, des vestiges fauniques et quelques dents humaines de Néandertaliens, mais malheureusement difficilement analysables de par la nature des fouilles anciennes (Callow in Callow \& Cornford, 1986). Des datations par thermoluminescence de l'ensemble II situé sous le III ont donné un âge moyen de $238000 \pm 35000$ ans BP, confirmant l'attribution de cet ensemble II au MIS 7 et la corrélation du III avec le MIS 6 (Huxtable in Callow \& Cornford, 1986). La faune de l'ensemble III étudiée par Scott (1986) comprend: Lepus sp., Canis lupus, Alopex lagopus, Ursus cf. spelaeus, Mammuthus primigenius, Coelodonta antiquitatis, Equus sp., Megaloceros giganteus, Cervus elaphus, Rangifer tarandus, Bison priscus et Rupicapra rupicapra. Ce cortège mammalien indique clairement un climat froid, continental et sec et un paysage très découvert de type steppique. La faune s'inscrit au sein de la biozone 24 , et témoigne d'une présence mammalienne durant le MIS 6 à La Cotte de Saint-Brelade alors que la plaine maritime autour de l'île était exondée.

\section{CAOURS (SOMME) (fig. $\left.2, \mathrm{n}^{\circ} 20\right)$}

Découvert à l'occasion de l'exploitation de carrière de tufs au début des années 1950 par L. Aufrère, le gisement de Caours situé dans la vallée du Scardon, affluent de la Somme, avait livré de l'industrie moustérienne associée avec des vestiges mammaliens qui avaient été attribués au dernier interglaciaire éemien (Breuil, 1952). La faune avait fait l'objet d'une étude par Patte qui avait déterminé (Patte, 1953 ; Breuil \& Barral, 1955): Cervus elaphus, Dama dama, Ursus sp., Equus sp. et Bos primigenius. Les nouvelles investigations menées sur les tufs du bassin de la Somme dans le cadre du programme CNRS «SITEP» (Eclipse II) sous la direction de P. Antoine depuis 2002 ont permis de mettre en évidence une formation de tufs calcaires reposant sur une nappe 
alluviale en position de très basse terrasse à Caours (Antoine et al., 2006). La séquence de tufs est séparée de la nappe alluviale périglaciaire sous-jacente par des limons fluviatiles calcaires se terminant par un sol de marais coiffé par un fin liseré de tourbe compactée. La séquence de tufs et la partie sommitale des limons fluviatiles contiennent une abondante faune malacologique tempérée qui permet de décrire une évolution climatique contemporaine des phases initiales d'un interglaciaire puis d'un optimum climatique correspondant au MIS 5e (Limondin-Lozouet in Antoine et al., 2006). La base du tuf comporte plusieurs horizons organiques qui ont livré des restes de mammifères et d'autres Vertébrés contemporains de l'optimum interglaciaire. Dans ces horizons, des campagnes de fouilles menées sur plusieurs centaines de mètres carrés ont permis la découverte de quatre niveaux du Paléolithique moyen en place, associant artefacts et grande faune. Une série de datations obtenues selon plusieurs méthodes indique un âge moyen centré autour de 123000 ans B.P. (Antoine et al., 2006; Bahain et al., sous-presse). Le gisement de Caours constitue un jalon essentiel et unique à ce jour qui permet de démontrer la présence humaine durant l'optimum climatique du MIS 5e en France septentrionale. La faune de Vertébrés (macro et méso faune) est encore en majeure partie inédite (Auguste in Antoine et al., 2006) et comprend un large spectre de taxons: Bos primigenius, Cervus elaphus, Dama cf. dama, Capreolus capreolus, Sus scrofa, Equus sp. de type caballin, Dicerorhinus hemitoechus (= Stephanorhinus hemitoechus), cf. Palaeoloxodon antiquus, Ursus arctos, Canis lupus, Aonyx antiqua, Castor fiber, Talpa cf. europea, cf. Emys orbicularis, oiseaux et amphibiens. La faune mammalienne se caractérise par un spectre uniquement constitué de formes de climat tempéré humide et indique un milieu dominé par la forêt mais où persistent des espaces découverts de type prairie ainsi que des zones aquatiques, confortant ainsi la mise en évidence de l'optimum climatique révélé par les mollusques et corrélé avec le MIS 5e aux environs de 123000 ans BP.

\section{LE ROZEL (MANCHE) (fig. 2, $\mathrm{n}^{\circ} 21$ )}

Le gisement du Rozel, situé sur la côte ouest de la presqu'île du Cotentin, a livré deux ensembles d'occupations anthropiques identifiés par des artefacts et des vestiges fauniques provenant de niveaux sableux dunaires sus-jacents à une plage fossile, initialement rattachés aux témoignages d'occupations du Paléolithique supérieur ancien (Scuvée \& Verague, 1984). Le réexamen de l'ensemble du contexte géomorphologique, sédimentaire, géochronologique, culturel et faunique a permis de montrer que la séquence sédimentaire couvre l'Eemien et le début glaciaire weichsélien, avec une série de datations OSL oscillant entre $115000 \pm 11000$ et $102000 \pm 10000$ ans BP, et que l'industrie lithique s'apparente en fait avec les cultures à débitage laminaire connues en Europe du Nord-Ouest durant le Weichsélien ancien et appartenant au Paléolithique moyen (Van VlietLanoë et al., 2006). Les deux ensembles d'occupations humaines du Rozel, celle de la Grande Dune et celle de l'Abri, ont tous deux livré du matériel faunique. Initialement déterminé par M.-F. Bonifay (in Scuvée \& Verague, 1984), le matériel étudié ne constituait en fait qu'une partie des vestiges mis au jour dans le gisement. Postérieurement à cette première publication, une pièce particulière avait été confiée pour détermination à L. Ginsburg qui identifia un nouveau taxon, en l'occurrence le morse, détermination confirmée ensuite par F. Poplin mais demeurée inédite. Cette pièce a été décrite et le taxon ajouté lors de l'étude de l'intégralité du matériel qui nous a permis de faire le point sur cette faune (Auguste in Van Vliet-Lanoë et al., 2006). La faune du Rozel comprend donc: Bos primigenius, Equus sp. de type caballin, Cervus elaphus, Megaloceros giganteus, Odobenus cf. rosmarus et un petit Carnivore. Cette faune indique un paysage en mosaïque de prairie parc-boisé, sous un climat tempéré. Le cheval s'apparente en particulier avec les formes identifiées durant les épisodes tempérés du début du Pléistocène supérieur (MIS $5 \mathrm{c}$ et 5a). La présence inhabituelle du morse en association avec cette faune tempérée n'est pas forcément incongrue, car d'une part cette espèce peut se rencontrer sous les mêmes latitudes durant l'Holocène, et d'autre part l'occupation humaine correspond avec un épisode tempéré moins marqué que les interglaciaires typiques. La faune s'inscrit bien au sein de la biozone 26 et caractériserait par ailleurs une des plus anciennes faunes connue pour le Weichsélien en France septentrionale, contemporaine du MIS 5c.

\section{LE MONT-DOL (ILLE-ET-VILAINE) (fig. 2, n² 22)}

Le célèbre gisement du Mont-Dol a été découvert en 1867 puis fouillé en 1872 par S. Sirodot, permettant la découverte de nombreux artefacts paléolithiques associés avec une très riche faune mammalienne caractérisée en particulier par une très abondante présence de dents et d'ossements de mammouths (Sirodot, 1873). La stratigraphie montre au-dessus d'une plage fossile attribuée à l'Eemien un dépôt contemporain d'un épisode froid qui serait corrélable avec le MIS 5d, surmonté par les niveaux archéologiques (Monnier, 1980). Des datations U-Th sur ossements donnent un âge de 106000 \pm 4000 ans BP (Laurent, 1993). La faune a fait l'objet d'une première analyse par Sirodot (1873), puis a été examinée par Simonet (Simonet \& Monnier, 1991). Les mégaherbivores (Proboscidien et Rhinocérotidé) ont été plus spécialement étudiés par Louguet (LouguetLefevre, 2005). Cette faune se compose de: Panthera spelaea, Panthera pardus, Canis lupus, Cuon sp., Vulpes vulpes, Ursus spelaeus, Ursus arctos, Sus scrofa, Cervus elaphus, Megaloceros giganteus, Rangifer tarandus, Dama dama, Capreolus capreolus, Bos primigenius, Bison priscus, Capra ibex, Mammuthus primigenius, Coelodonta antiquitatis, Equus germanicus, Equus hydruntinus, Marmota marmota, Meles meles. Il apparaît évident qu'une telle association faunique traduit très probablement un mélange d'au moins deux séries, l'une comportant un assemblage mammalien de contexte 
tempéré humide et de paysage de forêts et de prairies, l'autre étant caractérisée par un assemblage de taxons adaptés à un climat froid, continental et sec dans un milieu dominé par la steppe. Par contre, d'un point de vue biochronologique, les deux ensembles de faunes demeurent cohérents et caractérisent bien la biozone 26 , en contemporanéité avec au moins un épisode tempéré et un froid du Weichsélien ancien qui pourraient être corrélés avec les MIS $5 \mathrm{c}$ et $5 \mathrm{~b}$.

\section{BETTENCOURT-SAINT-OUEN (SOMME) (fig. 2, n² 23)}

La fouille du gisement de Bettencourt-Saint-Ouen, situé entre Amiens et Abbeville, a permis de mettre au jour cinq niveaux d'occupations humaines essentiellement représentés par des artefacts, au sein d'une séquence pédostratigraphique complexe (Locht, 2002). Tous ces niveaux paléolithiques se placent durant le début glaciaire weichsélien et couvrent les MIS 5d à 5a. Le niveau principal, N2b, est attribué au MIS 5a, entre 85000 et 75000 ans BP (Antoine in Locht, 2002). La quasi-totalité du matériel faunique provient du niveau N2b. Seules deux espèces ont été identifiées (Auguste, 2002): Equus sp. de type caballin et Bos primigenius. Le cheval s'apparente aux formes connues durant les périodes tempérées de la première partie du Pléistocène supérieur, il représente une forme intermédiaire entre le cheval éemien de Taubach, Equus taubachensis, et celui du MIS 3, Equus germanicus. Il traduit un paysage ouvert mais plutôt de type prairie que steppe sous un climat tempéré, en accord avec sa présence simultanée avec l'aurochs. La présence de cette faune au sein d'un niveau attribué au MIS 5a est donc cohérente.

\section{BEAUVAIS (OISE) (fig. $2, \mathrm{n}^{\circ} 24$ )}

Le gisement de la Justice à Beauvais a permis la découverte de deux niveaux d'occupations paléolithiques conservés au sein de sédiments sableux repris localement et re-déposés par transport aérien. Des vestiges lithiques et fauniques assez abondants y ont été extraits (Locht et al., 1995). Dans un premier temps, l'âge des dépôts avait posé problème d'un point de vue géologique et géochronologique, tandis que l'étude de la grande faune nous avait amené à la rapporter à une période froide du Pléistocène supérieur contemporaine du MIS 4 (Auguste in Locht et al., 1995). Par la suite, la corrélation avec le cadre régional des séquences loessiques (Antoine et al., 1998) et la datation par ESR et U-Th sur dents aux environs de 60000/40000 ans BP (Michel et al., 1999) ont permis de confirmer les interprétations biochronologiques et la mise en corrélation avec la fin du MIS 4 ou le début du MIS 3. La grande faune mammalienne comprend les espèces suivantes (Auguste in Locht et al., 1995; Auguste \& Patou-Mathis, 1999): Rangifer tarandus, Coelodonta antiquitatis antiquitatis, Equus sp. de type caballin, Mammuthus primigenius, Bison priscus, Mustela cf. eversmanni et Canis lupus. Cette faune est typique du cortège traditionnel de la «Steppe à Mammouth », elle témoigne bien en effet d'un climat nettement continental, froid et sec et d'un paysage de steppe. Le cheval présente en particulier une morphologie dentaire similaire à celle des chevaux des périodes froides du Pléistocène supérieur et diffère ainsi par exemple du cheval «tempéré» de Bettencourt. Cette association mammalienne caractérise bien la biozone 26 et s'accorde parfaitement avec l'âge attribué pour les deux niveaux archéologiques.

\section{AULT (SOMME) (fig. 2, $\mathrm{n}^{\circ} 25$ )}

Le gisement d'Ault-Onival, situé actuellement sous le niveau de la mer et accessible uniquement lors des plus basses mers, est connu depuis le début du XX $\mathrm{XX}^{\mathrm{e}}$ siècle. Il se caractérise par la présence d'une très grande quantité d'artefacts moustériens qui continuent à être extrait régulièrement par les collectionneurs (Agache, 1978). Le site a fait récemment l'objet d'un nouvel examen géomorphologique et stratigraphique, de prospection ainsi que de l'étude de vestiges fauniques demeurés inédits (Antoine \& Auguste, 2003). La formation continentale qui contient le niveau archéologique est constituée par un faciès de boue crayeuse à granules et abondants silex, préservée par un système de failles. Il s'agit de dépôts de versant formés alors que la falaise se situait à l'aplomb du site, durant une phase de retrait de la mer correspondant avec une période pléniglaciaire. Une étude de l'industrie lithique avait amené Perpère à rapporter la formation du site à la fin du Pléistocène moyen (Perpère, 1999). Les nouvelles analyses stratigraphiques et géomorphologiques montrent que la formation est contemporaine du Pléniglaciaire inférieur vers 55 000/ 50000 ans BP au début du MIS 3, interprétation confirmée par des dates obtenues par ESR/U-series sur dent de cheval qui sont de $55000 \pm 10000$ ans BP (Bahain et al., sous presse). La faune ne comprend que deux taxons (Antoine \& Auguste, 2003): Mammuthus primigenius, qui apparaît sous la forme typique de l'espèce et Equus sp. un Équidé de type caballin. Les adaptations écologiques de ces deux taxons sont en parfait accord avec l'âge pléniglaciaire du dépôt (climat continental froid et sec, paysage steppique) et le stade évolutif du mammouth est tout à fait compatible avec sa présence à Ault vers 55000 ans BP.

\section{HÉNIN-SUR-COJEUL (PAS-DE-CALAIS) (fig. 2, n² 26)}

Le gisement d'Hénin-sur-Cojeul, situé au sud d'Arras, a livré plusieurs séries d'artefacts et de vestiges fauniques au sein d'une séquence lœssique du Pléniglaciaire inférieur ou moyen. Plusieurs niveaux archéologiques ont été reconnus, certains directement formé en dépôt lœssique, d'autres au sein de pédocomplexes (Marcy et al., 1993). Des datations 14C ont donné des âges de $36600 \pm 1100$ et $37900 \pm 1800$ ans BP, indiquant plutôt des occupations durant le MIS 3. La faune provient de trois niveaux, F, G et $\mathrm{J}$, mais le cortège mammalien demeure globalement identique pour ces trois niveaux (Auguste in Marcy et al., 1993; Julien, 2004): Canis lupus, Vulpes vulpes, Alopex lagopus, Crocuta crocuta spelaea, Panthera spelaea, Equus sp. de type caballin, Coelodonta antiquitatis, Rangifer tarandus, Bison priscus, Mammuthus primige- 
nius et Marmota marmota. Cette association faunique est typique de celle de la «Steppe à Mammouth», avec des taxons tous adaptés à un climat continental froid et sec et à un paysage steppique. Elle est par ailleurs caractéristique de la biozone 26. La faune d'Hénin constitue l'une des dernières faunes connues pour le Pléniglaciaire weichsélien. Il faudra en effet attendre le Tardiglaciaire pour retrouver de nouveaux assemblages mammaliens bien datés en France septentrionale.

\section{DOURGES (PAS-DE-CALAIS) (fig. $2, \mathrm{n}^{\circ} 27$ )}

Après le hiatus correspondant au Pléniglaciaire supérieur où aucune série faunique n'a été découverte avec certitude en France septentrionale, c'est au cours du Tardiglaciaire durant le Bølling que les premiers témoignages du repeuplement mammalien vont être effectifs. Ainsi, dans le gisement de Dourges, au sud de Lille, une dépression lacustre dans le fond de la vallée de la Deûle a permis la mise en place et la conservation de sites du Paléolithique final livrant de l'industrie lithique et osseuse, ainsi que des vestiges de grands mammifères (Deschodt et al., 2005). La série sédimentaire comprend un épisode lacustre contemporain du Bølling, suivi par un assèchement durant le Dryas moyen, puis une phase de marécage au cours de l'Allerød. Le refroidissement du Dryas récent se marque par le début du comblement de la dépression par des alluvions limoneuses. Au moins trois occupations humaines notables ont été identifiées au sein de cette stratigraphie, un premier site de groupe à Federmesser durant le Bølling, une seconde halte de chasse Federmesser durant l'Allerød, puis une dernière présence paléolithique au début du Dryas récent. Des datations permettent de suivre très précisément cette histoire sédimentaire, avec un âge de $12430 \pm 90$ à $12230 \pm 70$ ans BP pour l'occupation du Bølling et de $11680 \pm 70$ ans BP pour celle de l'Allerød. Six espèces de grands mammifères sont présentes à Dourges (Auguste in Deschodt et al., 2005): Bos primigenius, Cervus elaphus, Equus cf. arcelini, Capreolus capreolus, Alces alces et Sus sp. L'aurochs est présent durant les trois périodes, avec en particulier un crâne dans le niveau Bølling; le chevreuil est également présent durant toutes les occupations; le cerf n'est présent que durant le Bølling et l'Allerød; le cheval n'est présent qu'à l'Allerød; un frontal d'élan a été découvert au début du Dryas récent. Pour le Bølling et l'Allerød, la faune indique un climat tempéré et un paysage de forêt ouverte, le Dryas récent se caractérisant par un climat froid. Cette faune présente à Dourges apporte ainsi de précieux renseignements sur les modalités de repeuplement du territoire immédiatement après le radoucissement du climat postérieur au dernier maximum glaciaire et met en évidence des phases de peuplements successifs observable au sein des associations fauniques. La faune est une des dernières représentantes de la biozone 26 .

\section{CONTY (SOMME) (fig. $2, \mathrm{n}^{\circ} 28$ )}

Le gisement de Conty, situé près d'Amiens, a livré une séquence sédimentaire de référence couvrant le Tardigla- ciaire ainsi qu'une importante occupation humaine du Paléolithique final rattachée au groupe à Federmesser. Il se situe au cœur de la plaine alluviale actuelle (Fagnart, 1997). La séquence sédimentaire est principalement représentée dans la zone archéologique par des dépôts de l'Allerød et du Dryas récent, mais des dépôts plus ancien datés du Bølling sont également présents latéralement. Outre l'industrie lithique et les vestiges fauniques, un bois de cerf décoré a également été découvert dans le niveau principal de Conty datée de l'Allerød. L'étude exhaustive de la faune, en cours de publication, a permis de mettre en évidence la présence des espèces suivantes (Auguste, sous presse a): Cervus elaphus, Capreolus capreolus, Bos primigenius et Equus cf. arcelini. Cette faune indique un climat tempéré et un paysage de forêts ouvertes non denses, en accord avec les autres approches naturalistes (Fagnart, 1997). Elle caractérise la fin de la biozone 26. Elle s'apparente très bien avec son homologue de Dourges.

\section{5 - DYNAMIQUE DES PEUPLEMENTS MAMMALIENS EN FRANCE SEPTENTRIONALE DURANT LE PLÉISTOCÈNE MOYEN ET SUPÉRIEUR: REPARTITION TEMPORELLE DES GROUPES TAXINOMIQUES}

L'inventaire (non exhaustif) des séries fauniques provenant des gisements pléistocènes du Nord de la France permet de réaliser une synthèse concernant la présence et les renouvellements au sein des différents groupes de taxons identifiés parmi les assemblages (fig. 3). Cet inventaire ne prend en compte que les grands mammifères, incluant les Rongeurs de forte taille ainsi que les Lagomorphes. Seuls les taxons bien identifiés et découverts en position stratigraphique interprétable ont été mentionnés. Globalement, ce sont 68 taxons qui ont été répertoriés et replacés au sein d'une échelle de lecture chronologique. Il apparaît ainsi une réelle paléobiodiversité mammalienne durant le Pléistocène en France septentrionale, mais celle-ci est en fait très variable suivant les périodes et les groupes zoologiques pris en considération.

Les Proboscidiens sont représentés par le groupe des mammouths et celui de l'éléphant de forêt. Le mammouth méridional est attesté par ses dernières formes avec au moins deux sous-espèces chronologiques, jusqu'au MIS 15. Dès le MIS 12, le mammouth des steppes est présent en France septentrionale mais demeure assez rare. Le mammouth laineux est identifié dès le MIS 8 à Tourville-la-Rivière (Lister, 1996), mais il ne devient courant au sein des faunes des périodes froides qu'à partir du MIS 6, et surtout durant le Pléistocène supérieur. L'éléphant antique est un animal très commun durant les phases tempérées et fait son apparition dès le MIS 15. Il n'est plus présent après l'Eemien.

Le rhinocéros étrusque évolué est présent jusqu'au MIS 15. La première apparition du rhinocéros de prairie est observée durant le MIS 9, l'espèce disparaissant de la 


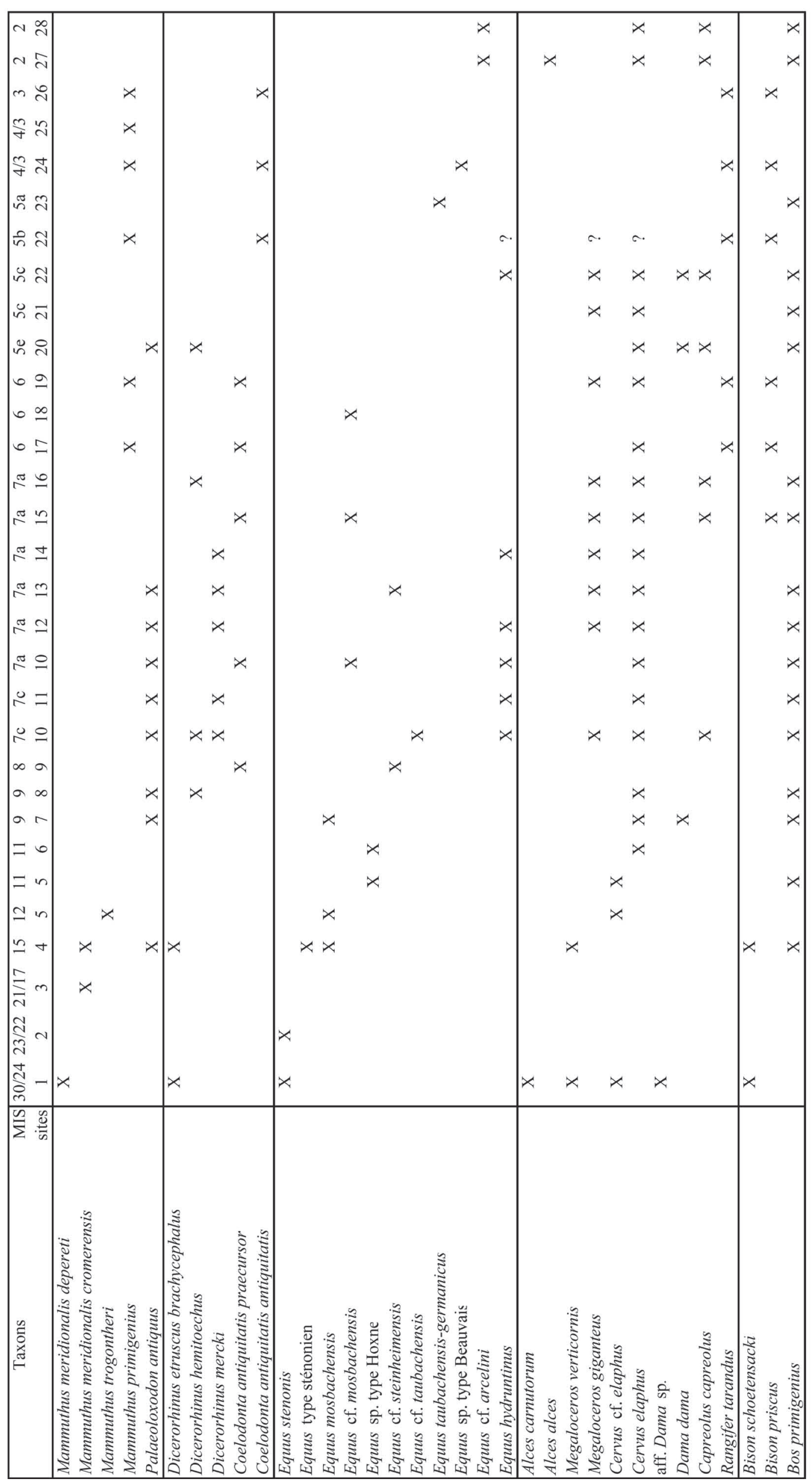




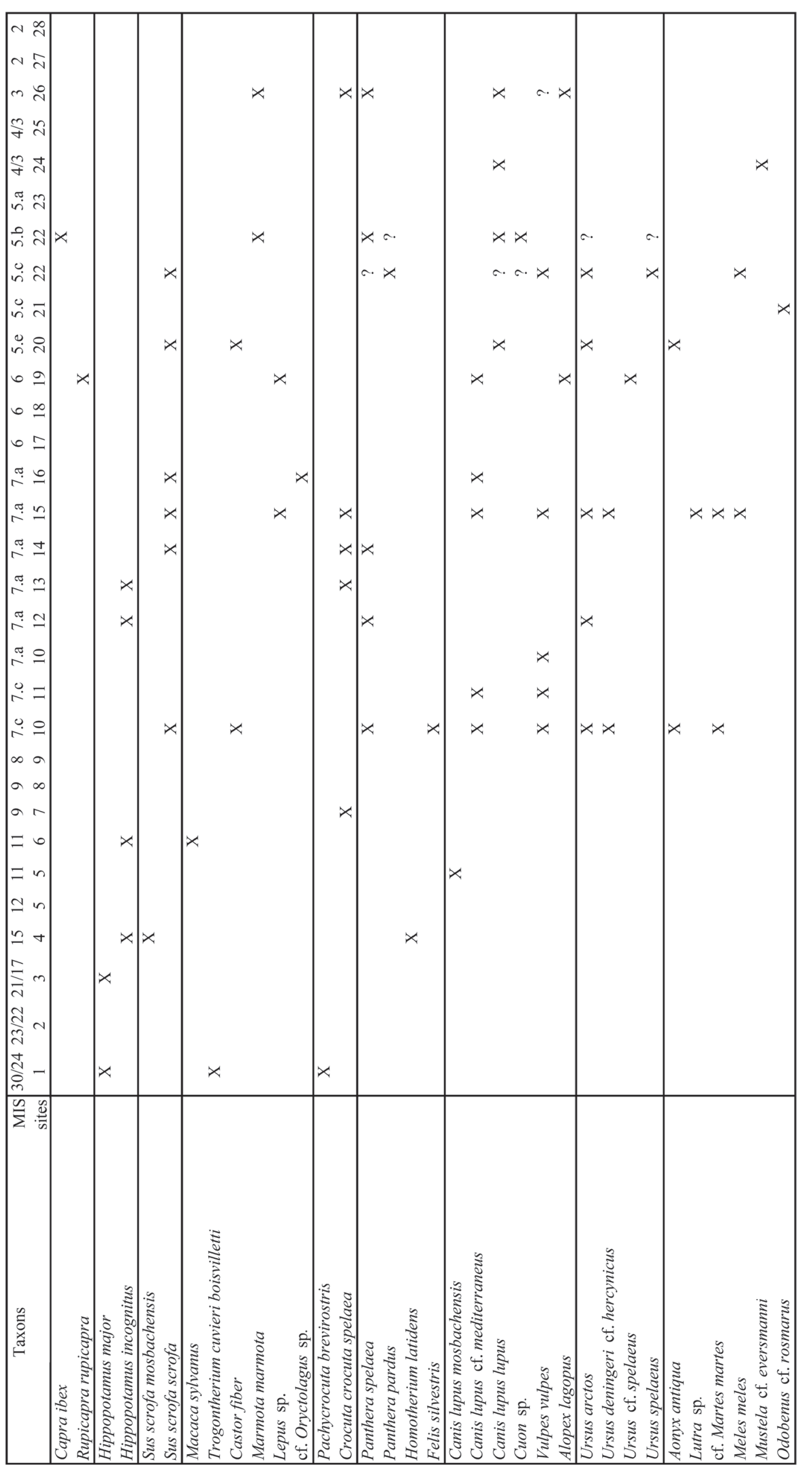

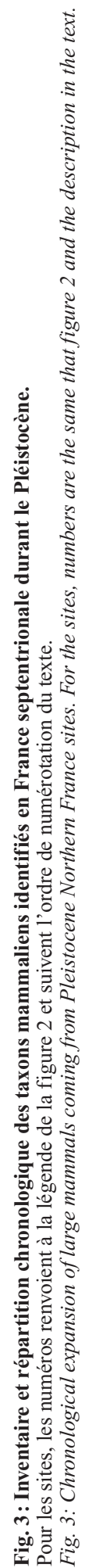


région après l'Eemien. Il ne se rencontre que durant les périodes tempérées. Le rhinocéros de Merck n'est curieusement présent dans le Nord de la France que durant les deux phases tempérées du MIS 7, laps de temps très restreint par rapport à son extension biochronologique importante (Guérin, 1980). Le rhinocéros laineux est identifié dès le MIS 8 sous sa forme archaïque et perdure ainsi jusqu'à la fin du MIS 6. Il est intéressant de noter qu'il est présent durant les périodes froides ou tempérées froides. Par contre le rhinocéros laineux typique ne se rencontre qu'à partir du MIS 5.b et uniquement en contexte glaciaire.

Les Équidés montrent la très grande variabilité connue pour le Pléistocène moyen et supérieur, avec la difficulté d'attribution taxinomique des différentes formes de chevaux. Le cheval de Sténon disparaît à la fin du Bavélien, mais des chevaux de type sténonien sont présents durant le Pléistocène moyen. Le cheval de Mosbach est identifié sous sa forme type du MIS 15 jusqu'au MIS 9, des chevaux proches de ce taxon lui sont ensuite attribués à la fin du Pléistocène moyen. Durant le MIS 11, un Cheval caballin de type tempéré est présent. Le Pléistocène supérieur se caractérise par la présence de plusieurs formes différentes en fonction du contexte écologique et de l'évolution du groupe taubachensis-germanicus, rejoignant ainsi le constat établi par Eisenmann et David (1994). Le dernier Équidé caballin identifié au Pléistocène supérieur en France septentrionale est l'Equus arcelini présent durant le Tardiglaciaire et proche de ses homologues du Bassin parisien (Bignon, 2008). E. hydruntinus est présent durant le MIS 7 et le MIS 5c. Il est absent des phases froides.

Les Cervidés sont également représentés par de nombreuses formes. Le groupe des élans est par contre très peu présent, avec l'élan de Saint-Prest à la fin du Pléistocène inférieur et l'élan actuel à la fin du tardiglaciaire. Le Mégacéros est assez courant à partir du MIS 7 jusqu'au MIS 5c. Le cerf élaphe est l'une des deux espèces les mieux représentées en France septentrionale dans le registre fossile, avec une première mention dès le MIS 11. Il se rencontre essentiellement durant les périodes tempérées, mais parfois en contexte glaciaire. Le daim est assez rare et ne se rencontre avec certitude qu'aux MIS 9, 5e et 5c, bien qu'une forme non définie soit présente auparavant à Saint-Prest durant le Bavélien (MIS 30/24). Il n'a pas été reconnu durant le MIS 7. Le chevreuil est présent durant les périodes tempérées de la fin du Pléistocène moyen, du début et de la fin du Pléistocène supérieur. Le renne n'est pas un animal courant. Il apparaît au MIS 6 seulement et disparaît avant le Tardiglaciaire.

Parmi les grands Bovidés, le bison des forêts est très rare et uniquement à la fin du Pléistocène inférieur et durant le MIS 15. Le bison des steppes apparaît au MIS $7 \mathrm{a}$ et se rencontre jusqu'au Tardiglaciaire. L'aurochs est le deuxième taxon le mieux représenté parmi les assemblages fossiles de France septentrionale. Il est identifié dès le MIS 15 et perdure jusqu'à la fin du Pléistocène supérieur. Il ne se rencontre uniquement que durant les périodes tempérées.
Les petits Bovidés sont extrêmement rares en France septentrionale, seuls le bouquetin et le chamois étant identifiés une fois dans deux gisements différents.

Deux espèces d'hippopotames sont présentes: le grand hippopotame au Bavélien et au début du Cromérien; l'H. incognitus, de taille plus modeste, identifié dès le MIS 15 et perdurant jusqu'au MIS 7a. Ce dernier est absent durant les MIS 9 et 7c, et dans l'état actuel des données durant l'Eemien.

Le sanglier est présent sous sa forme sub-spécifique archaïque durant le MIS 15, et sous sa forme type actuelle dès le MIS 7c jusqu'au MIS $5 \mathrm{c}$. Il est absent durant le Tardiglaciaire. On ne le rencontre que durant les phases tempérées.

Le macaque n'a été identifié qu'à La Celle durant le MIS 11.

Les grands Rongeurs et les Lagomorphes sont très rares parmi les accumulations fauniques pléistocènes de France septentrionale.

La hyène à face courte est présente à la fin du Pléistocène inférieur. Dès le MIS 9, la hyène des cavernes fait son apparition et ne sera identifiée ensuite que durant les MIS 7a et 3

Les Félidés sont aussi assez rares en France septentrionale.

Les Canidés sont mieux représentés, avec en particulier le phylum du loup dont on suit l'évolution depuis le MIS 11 avec la forme archaïque, en passant par le loup de la fin du Pléistocène moyen bien identifié au sein des gisements, jusqu'à la forme type de l'espèce connue dès l'Eemien et présente durant le Pléistocène supérieur.

Les Ursidés ne font leur apparition qu'à partir du MIS $7 \mathrm{c}$, avec essentiellement l'ours brun. Le phylum de l'ours des cavernes est représenté par la dernière forme évolutive de l'ours de Deninger (Auguste, 1995a) et présent sous sa forme type dans un seul gisement durant le MIS 5c.

Les petits Carnivores sont très rares.

Le morse n'a été identifié qu'au Rozel au début du Pléistocène supérieur.

Il apparaît clairement que la dynamique des peuplements mammaliens durant près d'un million d'années en France septentrionale va être étroitement tributaire de la modification des climats et des paysages. Les populations d'herbivores vont répondre de manière assez rapide, comme le montre par exemple la diversité des chevaux du Pléistocène supérieur, et de manière très nette. Les associations mammaliennes se caractérisent en effet par deux groupes majoritaires: le groupe des taxons adaptés aux paysages à dominante boisée mais avec présence constante d'espace de prairie durant les phases tempérées (par exemple: le sanglier, le chevreuil, les rhinocéros de forêt et de prairie, l'éléphant, l'aurochs,...); le groupe des paysages découverts steppiques durant les périodes froides (par exemple: le mammouth laineux, le rhinocéros laineux évolué, le renne, le bison des steppes,...). Il existe très peu d'associations mammaliennes de transition, excepté pour le MIS $7 \mathrm{a}$ où il est 
possible d'observer la mise en place d'un nouveau cadre écologique. Cette dichotomie bien marquée constitue un outil fiable qui, conjointement avec la caractérisation des stades évolutifs des différents taxons, permet de suivre avec précision la très importante dynamique de renouvellement des faunes dans le Nord de la France. Le biome de la «Steppe à Mammouth» n'apparaît clairement qu'à partir du MIS 6, mais il semble déjà présent durant le MIS 8. Il sera de nouveau identifiable dès le début du Weichsélien et disparaît définitivement avant le Tardiglaciaire. Ce sont donc essentiellement des spectres fauniques provenant des phases tempérées qui dominent les assemblages mammaliens en France septentrionale.

Un des apports particulièrement intéressant mis en exergue par cette étude est la présence maintenant assurée de deux phases tempérées identifiables au sein du MIS 7. En effet, lors de l'étude des séries fauniques provenant de Biache-Saint-Vaast (Auguste, 1995a), nous avions démontré que la faune des séries fluviatiles différait de celles des derniers niveaux, ces deux ensembles stratigraphiques étant par ailleurs séparés par un épisode froid (Tuffreau \& Sommé, 1988). Nous avions alors mis en corrélation le premier ensemble avec le MIS 7c et le second avec le MIS 7a. Cette interprétation permettait également de mieux comprendre le système des terrasses de la Somme et d'entériner la mise en évidence d'un double cycle glaciaire englobant le MIS 7 (8/7c et $7 b / 7 a)$ par Antoine (1990). L'évolution des données et des datations permet maintenant de confirmer ce scénario et cette subdivision au sein du MIS 7 est clairement identifiée dans plusieurs gisements (Lautridou et al., 2003; Bahain et al., 2007). La faune du MIS 7c, vers $230000 / 220000$ ans BP, se caractérise par la présence d'un cortège mammalien exclusivement tempéré, mais où l'hippopotame est curieusement absent. La faune contemporaine du MIS 7a, vers $200000 / 190000$ ans BP, reste globalement tempérée à tempérée froide, avec en particulier la présence de l'hippopotame, mais on note l'arrivée au sein du cortège du rhinocéros laineux sous sa forme archaïque et du bison des steppes, ainsi que de la hyène des cavernes. Le cheval est également différent entre les deux sous-stades isotopiques. Cette dichotomie a été également identifiée plus récemment en GrandeBretagne (Schreve, 2001a, 2001b).

\section{6 - DYNAMIQUE DE RENOUVELLEMENT DES ÉCOSYSTÈMES \\ EN FRANCE SEPTENTRIONALE DURANT LE PLÉISTOCÈNE MOYEN ET SUPÉRIEUR: L'APPORT DES GRANDS MAMMIFÈRES}

Il n'est pas dans notre propos de proposer un scénario global de l'évolution des systèmes écologiques depuis un million d'années dans le Nord de la France, ce travail dépassant très largement le cadre de cet article et nécessitant obligatoirement la mise en commun des données d'ordre géomorphologique, sédimentologique, et paléontologique (palynologie, petits mammifères, autres Verté- brés, malacologie, autres Invertébrés). Malgré tout, les résultats présentés et commentés précédemment offrent l'opportunité de proposer une modélisation partielle mais rendant compte des successions des différentes associations mammaliennes rencontrées au Pléistocène moyen et supérieur en France septentrionale.

La prise en compte des phénomènes migratoires évoqués supra couplant des mouvements spatiaux et temporels avec une évolution biologique variable suivant les taxons et les caractéristiques paléoécologiques des spectres fauniques qui se succèdent nous amènent à retracer pour quelques périodes clés les directions probables des flux fauniques vers la France septentrionale en particulier, mais valable à l'échelle de l'Europe du NordOuest (fig. 4). D'après ce scénario, en intégrant la fonction des deux «réservoirs » eurasiatiques pour les faunes tempérées et les faunes froides, l'Europe méditerranéenne et le Nord-Est asiatique, les mouvements des éléments fauniques au sein de ces successions d'écosystèmes traduisent bien des modifications majeures récurrentes, en terme d'alternance de climats continentaux, sec et froid avec des climats tempérés humides, et de paysages steppiques avec des milieux boisés à prairies herbeuses. Cette cyclicité n'est pas documentée, au moins d'après nos données, avant l'Elstérien au MIS 12, et s'arrête définitivement à la fin du Tardiglaciaire au Préboréal, au tout début de l'Holocène.

Si le modèle utilisé permet d'expliquer la raison de cette persistance récurrente des paramètres écologiques tout au long des différents cycles glaciaires concernés par le fonctionnement de ces deux zones refuges, il est plus délicat de comprendre pourquoi durant les périodes tempérées, les taxons inféodés aux espaces découverts sont toujours assez bien représentés. Deux réponses sont possibles: soit un fonctionnement différent du système climatique pléistocène par rapport à l'actuel; soit une régulation des milieux par les herbivores empêchant le développement total des zones forestières. Les travaux menés depuis quelques années sur l'évolution des écosystèmes pléistocènes apportent des éléments de réponse à cette question. Ainsi, il apparaît clairement que les biomes pléistocènes sont remarquablement stables durant plusieurs centaines de milliers d'années, en comparaison avec leurs homologues actuels, et qu'ils ne répondent pas directement aux variations cycliques des climats quaternaires, spécialement en domaine méditerranéen (Rodriguez, 2004). Cette stabilité globale des écosystèmes pléistocènes a été décrite sous le vocable de «continuité structurelle» par Miller (1996). Toujours en domaine méditerranéen, les travaux de Palombo (2007a, 2007b) permettent de préciser ce phénomène: il est observé une réponse des communautés mammaliennes aux changements majeurs du climat à très grande échelle chronologique, mais il ne semble pas y avoir par contre d'influences notables directes au niveau des cycles climatiques glaciaires/interglaciaires, les communautés d'herbivores en particulier ne montrent à cette échelle que des modifications liées à des phénomènes de compétitions alimentaires. Il en va de même pour les Carnivores qui ne répondent pas non plus à des contraintes 

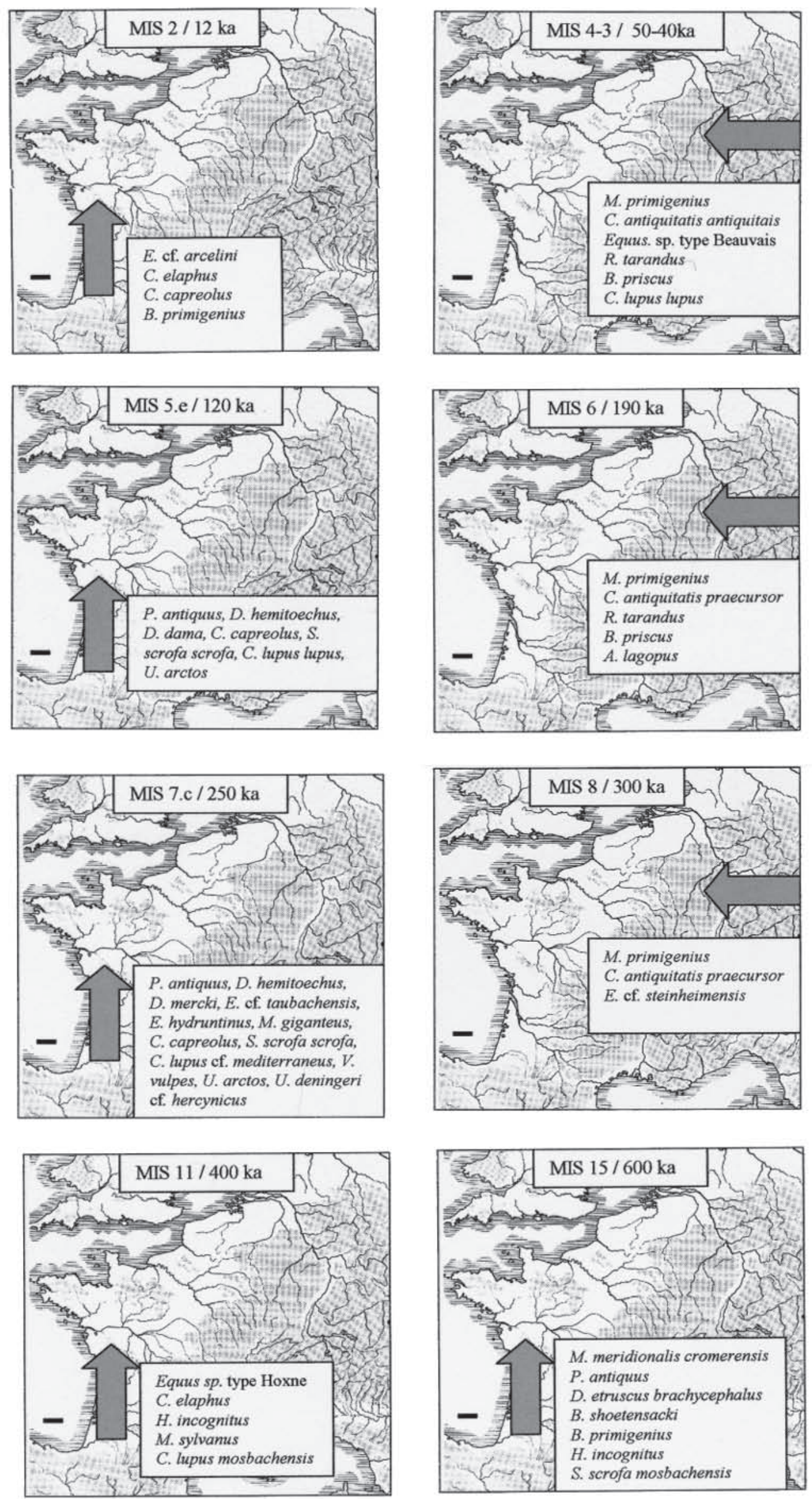

Fig. 4 : Dynamique spatio-temporelle des renouvellements mammaliens en Europe du Nord-Ouest (échelle: 100 kilomètres). Fig. 4: Dispersal events of large Mammals in North-Western Europe (scale: 100 kilometers). 
climatiques directes (Palombo et al., 2009). De manière très synthétique, les dynamiques de renouvellements fauniques au cours du Pléistocène moyen et supérieur en Europe correspondent donc plus à des rééquilibrages constants des communautés mammaliennes qu'à de profondes modifications structurelles. Les modalités de régulation d'un système évoquées supra par une activité importante des herbivores de toute taille sont évidentes lors de la présence de la «Steppe à Mammouth », mais celles-ci le demeurent également en contexte tempéré, même si les paramètres sont différents (animaux moins grégaires en particulier). Reste à expliquer dans cette dernière option pourquoi les mégaherbivores des périodes tempérés disparaissent également comme leurs homologues des périodes froides durant le Pléistocène supérieur et ne sont plus présents au Tardiglaciaire.

\section{7 - LE CADRE NORD-OUEST EUROPÉEN}

La comparaison des faunes mammaliennes pléistocènes de France septentrionale avec leurs homologues des contrées voisines situées en Europe du Nord-Ouest permet d'examiner les convergences et les éventuelles divergences de peuplement.

En Grande-Bretagne, les études concernant les grands Mammifères du Pléistocène moyen et supérieur ont permis d'aboutir à un cadre biochronologique cohérent, en particulier pour les dépôts situés en contexte fluviatile (Schreve et al., 2007). C'est en particulier après le MIS 12 que les séries fauniques permettent de suivre précisément les renouvellements fauniques corrélés avec les variations climatiques et environnementales, dont les associations ont été regroupées en «Mammal Assemblage- Zone» (Schreve, 1997, 2001a, 2001b; Schreve et al., 2007). Durant le MIS 11, plusieurs gisements livrent des spectres mammaliens très caractéristiques permettant la comparaison avec ceux découverts en France septentrionale. C'est le cas à Swanscombe, Clacton-onSea et Hoxne. Parmi cette faune, il est important de noter la présence de: Dicerorhinus hemitoechus, Dicerorhinus mercki, Cervus elaphus, Megaloceros giganteus, Capreolus capreolus, Dama dama clactoniana, Bos primigenius, Equus sp. de type caballin, Equus hydruntinus, Ursus deningeri, Macaca sylvanus et Palaeoloxodon antiquus. Ces faunes présentent des analogies avec celles identifiées à La Celle et Cagny-la-Garenne II, comme la présence du cerf, du cheval, de l'aurochs et du macaque, mais également des différences comme l'absence de l'éléphant et des rhinocéros dans le Nord de la France alors que l'hippopotame est lui absent en Angleterre. Durant le MIS 9, le site de Purfleet a livré un cortège mammalien composé en particulier de (Schreve et al., 2002): Macaca sylvanus, Crocuta crocuta spelaea, Palaeoloxodon antiquus, Equus sp., Dama dama, Cervus elaphus, Capreolus capreolus et Ursus arctos. Comme à Cagny-l'Épinette et Cléon, l'éléphant, le cerf, le daim et la hyène sont présents, alors qu'il n'y a pas de rhinocéros en Angleterre, ni d'aurochs et pas de macaque, de chevreuil et d'ours dans le Nord de la France. Par contre, dans les deux zones, l'hippopotame est absent, ainsi que le sanglier. En Angleterre, la dichotomie au sein du MIS 7 a également été reconnue, avec une première phase interglaciaire (MIS 7e ou 7c) mise en évidence dans le site d'Aveley et une faune dénommée «Ponds Farm MAZ» comprenant comme taxons caractéristiques Equus sp. et Palaeoloxodon antiquus (Schreve, 2001a, 2001b; Schreve et al., 2007). La seconde phase interglaciaire d'Aveley avec sa faune nommée «Sandy Lane MAZ» est plus riche en taxons spécifiques et a été reconnue également à Crayford et Ilford: Dicerorhinus mercki, Dicerorhinus hemitoechus, Equus sp., Bos primigenius, Cervus elaphus, Ursus arctos, Crocuta crocuta spelaea, Mammuthus trogontheri évolué et Coelodonta antiquitatis cf. praecursor. La première période tempérée au sein du MIS 7 est donc moins riche en Angleterre que dans le Nord de la France, mais la seconde montre une analogie claire, avec des taxons montrant des adaptations écologiques et des stades évolutifs similaires de part et d'autre de la Manche. Le dernier interglaciaire contemporain du MIS 5e n'est représenté en Angleterre que par des gisements paléontologiques, la présence humaine n'ayant pas à ce jour été démontrée durant cette période. Plusieurs gisements importants ont été découverts comme Trafalgar Square à Londres, Barrington et Joint Minor Cave, ce dernier donnant son nom à la MAZ (Currant \& Jacobi, 2001; Schreve et al., 2007). La faune comprend les principaux taxons suivants: Hippopotamus incognitus, Palaeoloxodon antiquus, Sus scrofa, Bos primigenius, Cervus elaphus, Dama dama, Dicerorhinus hemitoechus, Crocuta crocuta spelaea et Ursus arctos. Le seul site de France septentrional comparable, Caours, livre une faune quasiment équivalente, mais sans l'hippopotame (le Scardon n'a toutefois probablement pas eu une profondeur suffisante pour constituer une zone de prédilection de ces animaux) et la hyène (mais Caours est un site paléolithique où l'intervention des carnivores est très fugace).

La Grande-Bretagne montre donc une plus grande corrélation mammalogique avec le Nord de la France et sur une plus grande durée que ce qui peut s'observer avec l'Europe centrale par exemple (Schreve \& Bridgland, 2002).

Aux Pays-Bas, les gisements sont moins nombreux mais sont taxinomiquement aussi riches qu'en France septentrionale (Kolfschoten, 2001). Durant le Bavélien, le gisement de Noordzee II a livré les espèces suivantes: Mammuthus meridionalis, Equus bressanus, Dicerorhinus etruscus cf. brachycephalus, Hippopotamus major, Alces latifrons, cf. Bison menneri et cf. Praeovibos priscus. Cette faune est très proche de celle de SaintPrest et Grâce, mais également de celle de Wissant et caractérise bien la phase de transition entre les faunes villafranchiennes et les faunes du Pléistocène moyen. La faune du Maasvlakte I est attribuée au Cromérien et comprend en particulier: Ursus cf. deningeri, Матmu- 
thus meridionalis, Equus sp., Dicerorhinus etruscus brachycephalus, Sus scrofa, Hippopotamus major, Megaloceros verticornis et Cervus elaphus. Cette faune est proche de celle décrite dans la partie inférieure de la marne blanche de la carrière Carpentier et de la carrière Léon à Abbeville. Mis en contemporanéité avec le MIS 7, le gisement de Maastricht-Belvédère 4 a livré la faune suivante: Ursus sp., Equus sp., Dicerorhinus hemitoechus, Megaloceros giganteus, Cervus elaphus, Capreolus capreolus et cf. Bos primigenius. Ce spectre faunique s'apparente bien avec les séries mises en évidence pour les deux sous-stades 7c et 7a en France septentrionale. À Maastricht-Belvédère 5, la faune est contemporaine du début glaciaire weichsélien et est représentée par: Mammuthus primigenius, Equus sp., Coelodonta antiquitais, Cervus elaphus, Rangifer tarandus et cf. Bison priscus. Cette faune se rencontre dans le Nord de la France au Mont-Dol. La faune typique de la «Steppe à Mammouth » est présente dans les séries de Maasvlakte II et de Noordzee III durant les MIS 4/3 et se compose de: Canis lupus, Ursus spelaeus, Ursus arctos, Crocuta crocuta spelaea, Panthera spelaea, Mammuthus primigenius, Equus sp., Equus hydruntinus, Coelodonta antiquitatis, Megaloceros giganteus, Rangifer tarandus, Bison priscus et Ovibos moschatus.

La succession faunique observée durant le Pléistocène moyen et supérieur aux Pays-Bas s'avère donc presque identique à celle que nous mettons en évidence dans le Nord de la France, mais est moins continue de par l'absence de témoins pour certaines périodes.

Au-delà des différences ponctuelles notées entre les faunes anglaises et du Nord de la France qui nécessiteraient des travaux comparatifs entre les deux zones, le modèle de la dynamique des grands mammifères au sein de l'entité paléobiogéographique de l'Europe du NordOuest depuis la fin du Pléistocène inférieur jusqu'à la fin du Pléistocène supérieur apparaît très cohérent, se caractérisant par des composantes écologiques et biochronologiques similaires.

\section{8 - CONCLUSIONS}

L'évolution des associations mammaliennes en France septentrionale depuis la fin du Pléistocène inférieur jusqu'à la fin du Pléistocène supérieur, qu'il est possible de mettre en évidence à partir des nombreux gisements ayant livré des grands mammifères et du riche spectre taxinomique observé, permet donc de suivre assez bien les modifications majeures des climats et des milieux depuis environ un million d'années. Les grands mammifères s'avèrent être de bons outils biochronologiques, aptes à dater parfois très précisément les niveaux fossilifères et/ou paléolithiques d'où ils proviennent. Durant le Pléistocène moyen, c'est à partir du MIS 12 que les données sont les plus nombreuses et exploitables, avec un pic d'informations très net au cours du MIS 7. Cette phase globalement tempérée se caractérise ainsi par la présence de deux cycles glaciaires couvrant les MIS 8 /
$7 \mathrm{c}$ et $7 \mathrm{~b} / 7 \mathrm{a}$. La grande faune permet de témoigner de cette dichotomie avec une bonne résolution, et conforte les interprétations des géologues et géochronologues. Les grands renouvellements fauniques sont identifiés avec l'arrivée de nouveaux taxons ou de sous-espèces chronologiques dans la région, provenant soit de la zone méditerranéenne, soit de la zone nord-est européenne et nord-asiatique. L'alternance régulière des écosystèmes de type tempéré, marqués par des environnements mixtes de prairie parc-boisé, avec les écosystèmes de type froid, marqués par des paysages steppiques, est particulièrement visible et récurrente dans le Nord de la France depuis le MIS 12. Cette réponse parfois rapide et sans ambiguïté de la grande faune aux variations du cadre écologique offre ainsi l'opportunité de servir également de bon outil pour reconstituer les paramètres climatiques et environnementaux dans la zone considérée. La prise en compte du fonctionnement de ces biomes pléistocènes en intégrant les schémas de l'écologie systémique permet de replacer les entités fauniques au sein de ces systèmes complexes, où leur rôle de régulation du couvert végétal semble s'avérer essentiel. Le modèle décrit et présenté s'applique bien avec les proches zones géographiques situées au sein du même système écologique durant le Pléistocène comme en Grande-Bretagne et aux Pays-Bas.

\section{RÉFÉRENCES BIBLIOGRAPHIQUES}

AGACHE R., 1978 - Les ateliers paléolithiques d'Ault-Onival. In « $L a$ Somme pré-romaine et romaine». Amiens, Mémoire de la Société des Antiquaires de Picardie, 24, 74-77.

ANTOINE P., 1990 - Chronostratigraphie et environnement du Paléolithique du Bassin de la Somme. Villeneuve d'Ascq, Publications du C.E.R.P., 2, $231 \mathrm{p}$.

ANTOINE P., LAUTRIDOU J.P., SOMMÉ J., AUGUSTE P., AUFFRET J.P., BAIZE S., CLET-PELLERIN M., COUTARD J.-P., DEWOLF Y., DUGUÉ O., JOLY F., LAIGNEL B., LAURENT M., LAVOLLÉ M., LEBRET P., LECOLLE F., LEFEBVRE D., LIMONDIN-LOZOUET N., MUNAUT A.V., OZOUF J.C., QUESNEL F., \& ROUSSEAU D.-D., 1998 - Les formations quaternaires de la France du Nord-Ouest: Limites et Corrélations. Quaternaire, 9 (3), 227-241.

ANTOINE P., \& AUGUSTE P., 2003 - La paléofalaise et le site paléolithique d'Ault-Onival (Somme): données anciennes et nouvelles recherches. In J.-M. Hoeblich (dir), Les falaises de Picardie. Etats des lieux, enjeux, actions. Actes Colloque Amiens, avril 2001, 39-46.

ANTOINE P., AUGUSTE P., BAHAIN J.-J., COUDRET P. DEPAEPE P., FAGNART J.-P., FALGUĖRES C., FONTUGNE M., FRECHEN M., HATTÉ C., LAMOTTE A., LAURENT M., LIMONDIN-LOZOUET N., LOCHT J.-L., MERCIER N., MOIGNE A.-M., MUNAUT A.-V., PONEL P., \& ROUSSEAU D.D., 2003 - Paléoenvironnements pléistocènes et peuplements paléolithiques dans le bassin de la Somme (Nord de la France). Bulletin de la Société Préhistorique Française, 100 (1), 5-28.

ANTOINE P., LIMONDIN-LOZOUET N., AUGUSTE P., LOCHT J.-L., GALHEB B., REYSS J.-L., ESCUDÉ E., CARBONEL P., MERCIER N., BAHAIN J.-J., FALGUÈRES C., \& VOINCHET P., 2006 - Le tuf de Caours (Somme, France) : mise en évidence d'une séquence éemienne et d'un site paléolithique associé. Quaternaire, 17 (4), 281-320.

ANTOINE P., LIMONDIN-LOZOUET N., CHAUSSÉ C., LAUTRIDOU J.-P., PASTRE J.-F., AUGUSTE P., BAHAIN J.-J., FALGUÈRES C., \& GALEHB B., 2007 - Pleistocene fluvial terraces from northern France (Seine, Yonne, Somme): synthesis, and new results from interglacial deposits. Quaternary Science Reviews, 26, 2701-2723.

AUGUSTE P., 1988 - Etude des restes osseux des grands Mammifères des niveaux D, D1, IIbase. In A. Tuffreau \& J. Sommé (dir.), Le gisement paléolithique moyen de Biache-Saint-Vaast (Pas-de-Calais). 
Volume I. Paris, Mémoire de la Société Préhistorique Française, 21, 133-169.

AUGUSTE P., 1995a - Cadres biostratigraphique et paléoécologique du peuplement humain dans la France septentrionale durant le Pléistocène. Apports de l'étude paléontologique des grands mammifères de Biache-Saint-Vaast. Thèse de Doctorat, Muséum national d'Histoire naturelle, Paris, 5 vol., 1924 p.

AUGUSTE P., 1995b - Révision préliminaire des grands mammifères des gisements du Paléolithique inférieur et moyen de la vallée de la Somme. Bulletin de la Société Préhistorique Française, 92 (2), 143-154.

AUGUSTE P., \& PATOU-MATHIS M., 1999 - Beauvais (Oise): un nouveau gisement du Paléolithique moyen dans le Bassin parisien. In Actes du colloque international "The role of early Humans in the accumulation of European Lower and Middle Palaeolithic bone assemblages". Neuwied (1995), Monographie Romisch-Germanische Zentral-Museum Mainz, 42, 343-366.

AUGUSTE P., 2002 - Les vestiges fauniques. In J.L. Locht (dir.), Bettencourt-Saint-Ouen (Somme): Cinq occupations paléolithiques au début de la dernière glaciation. Paris, Maison des sciences de l'Homme, Documents d'Archéologie Française, 90, 39-47 et 136-137.

AUGUSTE P., CARPENTIER G., \& LAUTRIDOU J.-P., 2003 - La faune mammalienne de la basse terrasse de la Seine à Cléon (SeineMaritime, France): Interprétations taphonomiques et biostratigraphiques. Quaternaire, 14 (1), 5-14

AUGUSTE P., CLIQUET D., HERVIEU G., LIOUVILle M., LOUGUET S., MONNIER J.-L., \& RORIVE S., 2005 - Stratégies de subsistance dans 1'Ouest de la France au Pléistocène moyen et supérieur: acquisition et traitement des matières premières d'origines minérale et animale à Piégu (Côtes d'Armor), Ranville (Calvados) et au Mont-Dol (Ille et Vilaine). In N. Molines et al. (eds.), Les premiers peuplements en Europe. British Archaeological Reports International Series, Oxford, 1364, 519-532.

AUGUSTE P., 2008 - La faune. In D. Cliquet (dir.), Le site pléistocène moyen final de Ranville (Calvados-France) dans son contexte environnemental: analyse du fonctionnement d'une aire de boucherie soutirée par un réseau karstique. ERAUL, Liège, 119, 75-119.

AUGUSTE P., sous-presse a - La grande faune de Conty: Taxinomie, écologie et paléthnographie. In J.-P. Fagnart (dir.), La séquence tardiglaciaire et le gisement de Conty (Somme). Quaternaire hors série, Paris, 3, $23 \mathrm{p}$.

AUGUSTE P., sous-presse b - La faune de Gentelles. In A. Tuffreau (dir.), Le gisement pléistocène moyen de Gentelles (Somme, France). ERAUL, Liège, $9 \mathrm{p}$.

AULT DU MESNIL G. d', 1896 - Note sur le terrain quaternaire des environs d'Abbeville. Revue de l'École d'Anthropologie de Paris, 6 , 284-296.

BAHAIN J.-J., SARCIA M.N., FALGUÈRES C., \& YOKOYAMA Y., 1993 - Attempt at E.S.R. Dating of tooth enamel of French Middle Pleistocene sites. Applied Radiation and Isotopes, 44 (1-2), 267-272.

BAHAIN J.-J., 2007 - La méthode de datation par résonance de spin électronique (ESR) au Muséum national d'Histoire naturelle - Vingt ans de recherches méthodologiques et d'applications géochronologiques. Mémoire d'habilitation à diriger des recherches, Université Michel de Montaigne Bordeaux III, 88 p.

BAHAIN J.-J., FALGUÈRES C., LAURENT M., VOINCHET P., DOLO J.-M., ANTOINE P., \& TUFFREAU A., 2007 - ESR chronology of the Somme River Terrace system and first human settlements in Northern France. Quaternary Geochronology, 2, 356-362.

BAHAIN J.-J., GRUPPIONI G., FALGUÈRES C., \& DOLO J.-M., 2008 - Datation du remplissage du karst effectuée sur dents de Mammifères fossiles par les méthodes RPE/U-Th combinées. In D. Cliquet (dir.), Le site pléistocène moyen final de Ranville (CalvadosFrance) dans son contexte environnemental: analyse du fonctionnement d'une aire de boucherie soutirée par un réseau karstique. ERAUL, Liège, 119, 43-48.

BAHAIN J.-J., FALGUÈRES C., DOLO J.-M., ANTOINE P., AUGUSTE P., LIMONDIN-LOZOUET N., LOCHT J.-L., TUFFREAU A., TISSOUX H., \& FARKH S., sous-presse ESR/U-series dating of teeth recovered from well-stratifraphically age-controlled sequences from Northern France. Quaternary Geochronology, $12 \mathrm{p}$

BALESCU S., LAMOTHE M., \& LAUTRIDOU J.-P. , 1997 Luminescence evidence for two Middle Pleistocene interglacial events at Tourville, north-western France. Boreas, 26 (1), 61-72.

BALESCU S., \& TUFFREAU A., 2004 - La phase ancienne du Paléolithique moyen dans la France septentrionale (stades isotopiques 8 à 6): apports de la datation par luminescence des séquences loessiques. Archeologicheskii Almanach, 16, 5-22.

BEDEN M., \& DESCOMBES J.-C., 1982 - La faune mammalogique de Tourville-la-Rivière. In J.P. Lautridou (dir.), Le quaternaire de Normandie. Département Géologie UER Sciences, Rouen, 143-147.
BIGNON O., 2008 - Chasser les chevaux à la fin du Paléolithique dans le Bassin parisien. Stratégies cynégétiques et mode de vie au Magdalénien et à l'Azilien ancien. Oxford, Archaeopress, British Archaeological Reports International Series, volume 1747, $170 \mathrm{p}$.

BIQUAND D., 1974 - Position chronologique de la très haute nappe alluviale de Grâce (vallée de la Somme) par rapport à la limite paléomagnétique Brunhes-Matuyama. Bulletin de l'Association Française pour l'Étude du Quaternaire, 11, 157-159.

BOISVILLETTE M. de, 1849 - Lettre sur la découverte d'ossements à Saint-Prest (Eure-et-Loir). Bulletin de la Société Géologique de France, série 2, 6, 11-12.

BONIFAY M.F., 1969 - Relations entre les paléoclimats et les migrations des grands Mammifères quaternaires en Europe méridionale. Quaternaria, 11, 155-160.

BONIFAY M.F., 1990 - Relations between paleoclimatology and pliopleistocene biostratigraphic data in west european country. In E.H. Lindsay et al. (eds.), European Neogene Mammal Chronology. New York, Plenum Press, 475-485.

BONIFAY M.F., \& J.-P. BRUGAL, 1996 - Biogéographie et biostratigraphie des grandes faunes du Pléistocène inférieur et moyen en Europe du Sud: apport des gisements français. Paleo, 8, 19-30.

BOUCHUD J., 1964 - Présence d'Elephas meridionalis mutation cromerensis à Wissant (Pas-de-Calais). Bulletin de la Société d'Etudes et de Recherches Préhistoriques des Eyzies, 13, 1-4.

BOUCHUD J., 1968 - Étude sommaire de la molaire d'éléphant trouvée à Wissant (Pas-de-Calais). Revue anthropologique, 83-89.

BOURDIER F., 1969 - Étude comparée des dépôts quaternaires des bassins de la Seine et de la Somme. Bulletin d'Information des Géologues du Bassin de Paris, 21, 169-231.

BOURDIER F., CHALINE J., MUNAUT A.-V., \& PUISSEGUR J.-J., 1974a - La très haute nappe alluviale de la Somme. Bulletin de l'Association Française pour l'Étude du Quaternaire, 11, 137-143.

BOURDIER F., CHALINE J., MUNAUT A.-V., \& PUISSEGUR J.J., 1974b - Le complexe mindelien: II - La moyenne terrasse de l'Avre. Bulletin de l'Association Française pour l'Étude du Quaternaire, 11, 168-180.

BREUIL H., 1952 - Glanes conchyliologiques en France (Nord et SudOuest) déterminées par S. Kenard. Compte rendu de la XIII ${ }^{\mathrm{e}}$ Session du Congrès Préhistorique de France, Paris, 1950, Société Préhistorique Française, 191-240.

BREUIL H., \& BARRAL L., 1955 - Bois de Cervidés et autres os travaillés sommairement au Paléolithique ancien du vieux monde et au Moustérien des grottes de Grimaldi et de l'Observatoire de Monaco. Bulletin du Musée d'Anthropologie Préhistorique de Monaco, 2, 3-31.

BRÜNING H., 1980 - Die eiszeitliche Tierwelt von Mosbach. Ihre Umwelt. Ihre Zeit. Naturhistorische Museum Mainz, Museumsfürher, $6,60 \mathrm{p}$.

CALLOW P., \& CORNFORD J.M. (eds.), 1986 - La Cotte de SaintBrelade 1961-1978. Excavations by C.B.M. Mc Burney. Geobooks, Cambridge, $433 \mathrm{p}$

CLIQUET D. (dir), 2008 - Le site pléistocène moyen final de Ranville (Calvados-France) dans son contexte environnemental: analyse $d u$ fonctionnement d'une aire de boucherie soutirée par un réseau karstique. ERAUL, Liège, 119, $211 \mathrm{p}$.

COMMONT V., 1910 - Les gisements paléolithiques d'Abbeville. Excursion de la Société Géologique du Nord, Faculté des Sciences de Lille, Abbeville, 1910, Annales de la Société Géologique du Nord, 39, 249-292.

COMMONT V., 1912 - Moustérien à faune chaude dans la Vallée de la Somme à Montières-lès-Amiens. Congrès International d'Archéologie et d'Anthropologie Préhistorique, compte-rendu $14^{e}$ session, Genève, 1912, 291-300.

CURRANT A.P., \& JACOBI R.M., 2001 - A formal mammalian biostratigraphy for the Late Pleistocene of Britain. Quaternary Science Reviews, 20, 1707-1716.

DELPECH F., 1999 - Biomasse d'Ongulés au Paléolithique et inférences sur la démographie. Paléo, 11, 19-42.

DESCHODT L., TEHEUX E., LANTOINE J., AUGUSTE P., \& LIMONDIN-LOZOUET N., 2005 - L'enregistrement tardiglaciaire de Dourges (Nord de la France, bassin de la Deûle): évolution d'une zone lacustre et gisements archéologiques associés. Quaternaire, 16 (3), 229-252.

DESCOMBES J.-C., 1980 - La première faune rissienne de la basse vallée de la Seine. Implications biostratigraphiques et paléoécologiques. Thèse $3^{\mathrm{e}}$ cycle, Université de Poitiers, $205 \mathrm{p}$.

DESCOMBES J.-C., 1982 - Tourville-la-Rivière (Seine-Maritime) : le premier grand gisement de mammifères du Pléistocène moyen (Riss) dans la basse vallée de la Seine. Compte-rendus de l'Académie des Sciences, Paris, 295, série II, 905-907.

DESCOMBES J.-C., 1983 - Etude paléontologique du gisement pléistocène moyen de Tourville-la-Rivière (Seine-Maritime, France) 
Bulletin de l'Association Française pour l'Étude du Quaternaire, série 2, 16, 161-169.

DESCOMBES J.C., \& CARPENTIER G., 1987 - La faune de Grands Mammifères de Tourville-la-Rivière. Bulletin du Centre de Géomorphologie du CNRS, 32, 19-23.

EISENMANN V., 1991 - Les chevaux quaternaires européens (Mammalia, Perissodactyla). Taille, typologie, biostratigraphie et taxonomie. Geobios, 24, 747-759.

EISENMANN V., \& DAVID F., 1994 - Le cheval de Mauran (HauteGaronne): stade de transition évolutive entre E. taubachensis et E. germanicus? In C. Farizy, F. David \& J. Jaubert (dir.), Hommes et Bisons du Paléolithique moyen à Mauran (Haute-Garonne). Supplément à Gallia Préhistoire, Paris, 30, 41-46.

FAGNART J.-P., 1997 - La fin des temps glaciaires dans le Nord de la France. Mémoire de la Société Préhistorique Française, Paris, Société Préhistorique Française, 24, 270 p.

FAURE M., 1983 - Les Hippopotamidae (Mammalia, Artiodactyla) d'Europe occidentale. Thèse $3^{\mathrm{e}}$ cycle, Université Claude-Bernard Lyon 1, $233 \mathrm{p}$.

FAURE M., 1984 - Hippopotamus incognitus nov. sp., un hippopotame (Mammalia, Artiodactyla) du Pléistocène d'Europe occidentale. Geobios, 17 (4), 427-434.

FAURE M., 1985 - Les Hippopotames quaternaires non-insulaires d'Europe occidentale. Nouvelles Archives du Muséum d'Histoire Naturelle de Lyon, 23, 13-79.

GIBBARD P., \& COHEN K.M., 2008 - Global chronostratigraphical correlation table for the last 2.7 million years. Episodes, 31 (2), 243-247.

GUERIN C., 1980 - Les Rhinocéros (Mammalia, Perissodactyla) du Miocène terminal au Pléistocène supérieur en Europe occidentale. Comparaison avec les espèces actuelles. Documents des Laboratoires de Géologie de Lyon, 79 (1-3), 1-1185.

GUÉRIN C., 1990 - Biozones or Mammal Units? Methods and Limits in Biochronology. In E.H. Lindsay et al. (eds.), European Neogene Mammal Chronology. Plenum Press, New-York, 119-130.

GUÉRIN C., 1998 - Mammifères, datations et paléoenvironnements en préhistoire. Quaternaire, 9 (4), 249-260.

GUÉRIN C., DEWOLF Y., \& LAUTRIDOU J.-P., 2003 - Révision d'un site paléontologique célèbre: Saint-Prest (Chartres, France). Geobios, 36, 55-82.

GUÉRIN C., 2004 - Les rhinocéros (Mammalia, Perissodactyla) du gisement villafranchien moyen de Saint-Vallier (Drôme). In M. Faure \& C. Guérin (eds.), Le gisement pliocène final de SaintVallier (Drôme, France). Geobios, MS 26, 259-278.

GUÉRIN C., 2007 - Biozonation continentale du Plio-Pléistocène d'Europe et d'Asie occidentale par les Mammifères : état de la question et incidence sur les limites Tertiaire/Quaternaire et Plio/Pléistocène. Quaternaire, 18 (1), 23-33

GUIPERT G., 2005 - Reconstitution et position phylétique des restes crâniens de l'Homme de Tautavel (Arago 21-47) et de Biache-SaintVaast 2. Apports de l'imagerie et de l'analyse tridimensionnelle. Thèse de Doctorat, Université Paul Cézanne Aix-Marseille III, 463 p.

GUTHRIE R.D., 1990 - Frozen fauna of the mammoth steppe: the story of Blue Babe. University of Chicago Press, Chicago, $323 \mathrm{p}$.

HAESAERTS P., \& DUPUIS C., 1986 - Contribution à la stratigraphie des nappes alluviales de la Somme et de l'Avre dans la région d'Amiens. In "Chronostratigraphie et faciès culturels du Paléolithique inférieur et moyen dans l'Europe du Nord-Ouest». Supplément Bulletin de 1'Association Française pour l'Étude du Quaternaire, 25-26, 171-186.

HALLEGOUËT B., MONNIER J.-L., \& GAGNEPAIN J., 1993 Le site paléolithique moyen de Piégu (en Pléneuf-Val-André). Premiers résultats de fouilles. Mémoire de la Société d'Émulation des Côtes-d'Armor, 121, 3-17.

HEINZELIN J. de, 1964 - Cailloutis de Wissant, capture de Marquise et percée de Warcove. Bulletin de la Société Belge de Géologie, Paléontologie et Hydrogéologie, 73, 141-161.

JULIEN M.-A., 2004 - Étude archéozoologique du site d'Hénin-surCojeul (Pas-de-Calais, France). Mémoire de DEA, Muséum national d'Histoire naturelle Paris, 93 p.

KAHLKE R.-D., 1999 - The history of the origin, evolution and dispersal of the Late Pleistocene Mammuthus-Coelondonta faunal complex in Eurasia (large Mammals). Rapid City, 219 p.

KAHLKE R.-D., 2007 - Late Early Pleistocene European large mammals and the concept of an Epivillafranchian biochron. In R.-D. Kahlke, L. Maul \& P.P.A. Mazza (eds.), Late Neogene and Quaternary biodiversity and evolution: Regional developments and interregional correlations. Volume II. Frankfurt am Main, Courier Forschungsinstitut Senckenberg, 259, 265-278.

KOLFSCHOTEN T. Van, 1992 - Aspects of the migration of Mammals to Northwestern Europe during the Pleistocene, in parti- cular the reintegration of Arvicola terrestris. In W. von Koenigswald \& L. Werdelin (eds.), Mammalian Migration and Dispersal Events in the European Quaternary. Frankfurt am Main, Courier Forschungsinstitut Senckenberg, 153, 213-220.

KOLFSCHOTEN T. Van, 1995 - On the application of fossil mammals to the reconstruction of the palaeoenvironment of northwestern Europe. Acta Zoologica Cracoviensia, 38, 73-84.

KOLFSCHOTEN T. Van, 2001 - Pleistocene Mammals from the Netherlands. Bolletino della Societa Paleontologica Italiana, 40 (2), 209-215.

LAMOTTE A.,AUGUSTE P., LOCHT J.-L., TUFFREAU A., 2005 L'acquisition des ressources minérales et animales au Paléolithique inférieur et moyen dans le nord de la France dans leur contexte écologique: état des recherches récentes. In N. Molines et al. (eds.), Les premiers peuplements en Europe. British Archaeological Reports International Series, Oxford, 1364, 545-554.

LAURENT M., 1993 - Datation par résonance de spin électronique (ESR) de quartz de formations quaternaires: comparaison avec le paléomagnétisme. Thèse de Doctorat, Muséum national d'Histoire naturelle Paris, $103 \mathrm{p}$.

LAUTRIDOU J.-P., AUFFRET J.-P., LÉCOLLE F., LEFÈVRE D., LERICOLAIS G., ROBLIN-JOUVE A., BALESCU S., CARPENTIER G., CORDY J.-M., DESCOMBES J.-C., OCCHIETTI S., \& ROUSSEAU D.-D., 1999 - Le fleuve Seine, le fleuve Manche. Bulletin de la Société Géologique de France, 170, 545-558.

LAUTRIDOU J.-P., 2003 - La datation du Quaternaire normand: tableau des éléments de datation et de la chronostratigraphie. Quaternaire, 14 (1), 65-71.

LAUTRIDOU J.-P., AUGUSTE P., CARPENTIER G., CORDY J.-M., LEBRET P., LECHEVALIER C., \& LEFEBVRE D., 2003 - L'Eemien et le Pléistocène moyen récent fluvio-marin et continental de la vallée de la Seine de Cléon au Havre (Normandie). Quaternaire, 14 (1), 25-30.

LEGENDRE S., 1989 - Les communautés de mammifères du Paléogène (Éocène supérieur et Oligocène) d'Europe occidentale: structures, milieux et évolution. Münchner Geowissenschaftliche Abhandlungen, (A), 16, 1-110.

LIMONDIN-LOZOUET N., ANTOINE P., AUGUSTE P., BAHAIN J.-J., CARBONEL P., CHAUSSÉ C., CONNET N., DUPÉRON J., DUPÉRON M., FALGUÈRES C., FREYTET P., GHALEB B., JOLLY-SAAD M.C., LHOMME V., LOZOUET P., MERCIER N., PASTRE J.-F., \& VOINCHET P., 2006 - Le tuf calcaire de la Celle-sur-Seine (Seine et Marne): nouvelles données sur un site clé du stade 11 dans le Nord de la France. Quaternaire, 17 (2), 5-29.

LIOUVILLE M., 2007 - La variabilité morphologique du Cerfélaphe au Pléistocène moyen et supérieur. Aspects biologiques, écologiques et cynégétiques. Thèse de Doctorat, Muséum national d'Histoire naturelle, Paris, $408 \mathrm{p}$.

LISTER A.M., 1992 - Mammalian fossils and Quaternary biostratigraphy. Quaternary Science Review, 11, 329-344.

LISTER A.M., 1996 - Evolution and taxonomy of Eurasian mammoths. In J. Shoshani \& P. Tassy (eds.), The Proboscidea. Evolution and Palaeoecology of Elephants and their relatives. Oxford University Press, Oxford, 203-213.

LISTER A.M., 1997 - The evolutionary response of vertebrates to Quaternary environmental change. NATO ASI Series, I, 47, 287-302.

LISTER A.M., 2004 - The impact of Quaternary Ice Ages on mammalian evolution. Philosophical Transactions of the Royal Society of London, B, 359, 221-241.

LOCHT J.-L., SWINNEN C., ANTOINE P., AUGUSTE P., PATOUMATHIS M., DEPAEPE P., FALGUERES C., LAURENT M., BAHAIN J.-J., \& MATHYS P., 1995 - Le gisement paléolithique moyen de Beauvais (Oise). Bulletin de la Société Préhistorique Française, 92 (2), 213-226.

LOCHT J.-L. (dir.), 2002 - Bettencourt-Saint-Ouen (Somme). Cinq occupations paléolithiques au début de la dernière glaciation. Documents d'Archéologie Française, Éditions de la Maison des sciences de l'Homme, Paris, 90, 176 p.

LOUGUET-LEFEVRE S., 2005 - Les mégaherbivores (Éléphantidés et Rhinocérotidés) au Paléolithique moyen en Europe du NordOuest. Paléoécologie, taphonomie et aspects palethnographiques. British Archaeological Records International Series, Archaeopress, Oxford, 1451, p. 1-267.

MARCY J.-L., AUGUSTE P., FONTUGNE M., MUNAUT A.-V., \& VAN VLIET LANOE B., 1993 - Le gisement moustérien d'Héninsur-Cojeul (Pas-de-Calais). Bulletin de la Société Préhistorique Française, 90 (4), 251-256.

MICHEL V., LOCHT J.-L., ANTOINE P., MASAOUDI H., FALGUËRES C., \& YOKOYAMA Y., 1999 - Datation ESR/U-Th combinée des restes fauniques du site moustérien de plein air de Beauvais (France). Compte Rendu de l'Académie des Sciences, Sciences de la Terre et des planètes, Paris, 329, 369-375. 
MILLER III W., 1996 - Ecology of coordinate stasis. Paleogeography, Paleoclimatology, Paleoecology, 127, 177-190.

MOIGNE A.-M., 1988 - Les faunes du gisement de Cagny-l'Épinette. In Actes Colloque «Cultures et industries lithiques en milieu loessique». Revue Archéologique de Picardie, Amiens, 1986, 1-2, 69-71.

MOIGNE A.-M., 1989 - Cagny-l'Épinette: la grande faune. In A. Tuffreau (dir.), Livret-guide de l'excursion dans la vallée de la Somme. Colloque international "L'Acheuléen dans l'Ouest de l'Europe», Saint-Riquier, 1989, 82-87.

MONNIER J.-L., 1980 - Le Paléolithique de la Bretagne dans son cadre géologique. Travaux du laboratoire d'Anthropologie Préhistorique de Rennes, $607 \mathrm{p}$

MONNIER J.-L., 1991 - La Préhistoire de Bretagne et d'Armorique. J.P. Gisserot, Rennes, 123 p.

MOULLÉ P.-E., 1997-1998 - Les grands Mammifères de la grotte du Vallonnet (Roquebrune-Cap-Martin, Alpes-Maritimes). Synthèse des études antérieures et nouvelles déterminations. Bulletin $d u$ Musée d'Anthropologie Préhistorique de Monaco, 39, 29-36.

MUNAUT A.-V., 1989 - Analyse palynologique de la coupe de la carrière Carpentier à Abbeville. In A. Tuffreau (dir.), Livret-guide de l'excursion dans la vallée de la Somme. Colloque international "L'Acheuléen dans l'Ouest de l'Europe», Saint-Riquier, 1989, 11-14.

OWEN-SMITH R.N., 1988 - Megaherbivores. The influence of very large body size in ecology. Cambridge University Press, Cambridge, $392 \mathrm{p}$.

PALOMBO M.R., 2007a - Climate change versus biotic interaction: a case study of large mammal faunal complexes on the Italian Peninsula from the Pliocene to the Late Pleistocene. New methodological approaches. In R.-D. Kahlke, L. Maul \& P.P.A. Mazza (eds.), Late Neogene and Quaternary biodiversity and evolution: Regional developments and interregional correlations. Volume II. Frankfurt am Main, Courier Forschungsinstitut Senckenberg, 259, 13-46.

PALOMBO M.R., 2007b - What is the boundary for the quaternary period and pleistocene epoch? The contribution of turnover patterns in large mammalian complexes from north-western Mediterranean to the debate. Quaternaire, 18 (1), 35-53.

PALOMBO M.R., \& R. SARDELLA, 2007 - Biochronology and biochron boundaries: A real dilemma or a false problem? An example based on the Pleistocene large mammalian faunas from Italy. Quaternary international, 160, 30-42.

PALOMBO M.R, ALBERDI M.T., AZANZA B., GIOVINAZZO C., PRADO J.L., \& SARDELLA R., 2009 - How did environmental disturbances affect carnivoran diversity? A case study of the Plio-Pleistocene Carnivora of the North-Western Mediterranean. Evolutionary Ecology, 23, 569-589.

PATTE E., 1924 - Contribution à l'étude du Quaternaire dans la vallée de l'Oise. Bulletin de la Société Géologique de France, série 4, 24 (7-8), 483-514

PATTE E., 1941 - Les enseignements de la vallée de la Charente. $\mathrm{La}$ revue Scientifique, 79 (4), 216-222.

PATTE E., 1953 - Remarques sur quelques Daims fossiles. Bulletin de la Société Géologique de France, série 6, 3 (7-8), 657-666.

PATTE E., 1967 - La basse terrasse de Sempigny (Oise) : ses industries osseuses et lithiques, sa faune. L'Anthropologie, 71 (5-6), 401-434

PERPÈRE M., 1999 - Le débitage Levallois d'Ault. L'Anthropologie, 103 (3), 343-376.

PONTIER G., 1910 - Remarques sur les faunes d'Abbeville. Annales de la Société Géologique du Nord, 39, 293-303.

PONTIER G., 1914 - Étude sur l'Elephas primigenius de la vallée de l'Aa. Annales de la Société Géologique du Nord, 43, 30-89.

PONTIER G., 1928 - Les Éléphants fossiles d'Abbeville. Annales de la Société Géologique du Nord, 53, 20-46.

POPLIN F., 1969 - Étude des restes osseux provenant de la carrière de Wissant. Septentrion, 1, 69-72.

PRAT F., 1968a - Recherches sur les Équidés pléistocènes en France. Thèse de Doctorat d'État, Université de Bordeaux, 4 tomes, 663 p.

PRAT F., 1968b - Observations sur quelques ossements découverts dans la basse terrasse de l'Oise à Moru, Commune de Rhuis, Oise. In «La Préhistoire: problèmes et tendances». Colloque International CNRS, Paris, 337-348.

PRAT F., 1980 - Les Équidés villafranchiens en France. Genre Equus. Cahiers du Quaternaire, 2, 290p.

RODRIGUEZ J., 2004 - Stability in Pleistocene Mediterranean mammalian communities. Paleogeography, Paleoclimatology, Paleoecology, 2007, 1-22.

ROUGIER H., 2003 - Étude descriptive et comparative de BiacheSaint-Vaast 1 (Biache-Saint-Vaast, Pas-de-Calais, France). Thèse de Doctorat, Université Bordeaux 1, 424 p.

SHREVE D., 1997 - Mammalian biostratigraphy of the later Middle Pleistocene in Britain. Thèse, Université de Londres, 776 p.
SCHREVE D.C., 2001a - Differentiation of the British late Middle Pleistocene interglacials: the evidence from mammalian biostratigraphy. Quaternary Science Reviews, 20, 1963-1705.

SCHREVE D.C., 2001b - Mammalian evidence from Middle Pleistocene fluvial sequences for complex environmental change at the oxygen isotope substage level. Quaternary International, 79 65-74.

SCHREVE D.C., \& BRIDGLAND D.R., 2002 - Correlation of English and German Middle Pleistocene fluvial sequences based on mammalian biostratigraphy. Netherlands Journal of Geosciences / Geologie en Mijnbouw, 81, 357-373.

SCHREVE D.C., BRIDGLAND D.R., ALLEN P., BLACKFORD J.J., GLEED-OWEN C.P., GRIFFITHS H.I., KEEN D.H., \& WHITE M.J., 2002 - Sedimentology, palaeontology and archaeology of late Middle Pleistocene River Thames terrace deposits at Purfleet, Essex, UK. Quaternary Science Reviews, 21, 1423-1464.

SCHREVE D.C., KEEN D.H., LIMONDIN-LOZOUET N., AUGUSTE P., SANTISTEBAN J.I., UBILLA M., MATOSHKO A., BRIDGLAND D.R., \& WESTAWAY R., 2007 - Progress in faunal correlation of Late Cenozoic fluvial sequences 200-4: the report of the IGCP 449 Biostratigraphy subgroup. Quaternary Science Reviews, 26, 2970-2995.

SCOTT K., 1986 - The large mammal fauna. In P. Callow \& J.M. Cornford (eds.), La Cotte de Saint-Brelade 1961-1978. Excavations by C.B.M. Mc Burney. Geobooks, Cambridge, 109-137.

SCUVÉE F., \& VERAGUE J., 1984 - Paléolithique supérieur en Normandie occidentale: l'abri sous-roche de la pointe du Rozel (Manche). Cherbourg, LITTUS-C.E.H.P., 150 p.

SIMONET P., \& MONNIER J.-L., 1991 - Approche paléoécologique et taphonomique de la grande faune du gisement moustérien du Mont-Dol (Ille-et-Vilaine, France). Quaternaire, 2 (1), 5-15.

SIRODOT S., 1873 - Fouilles exécutées au Mont-Dol (Ille-et-Vilaine) en 1872. Mémoires de la Société d'Émulation des Côtes-du-Nord, 11, 59-108.

SOMMÉ J., \& TUFFREAU A., 1976 - Les formations pléistocènes de Wissant (Pas-de-Calais). In "Livret-Guide excursion A10: NordOuest de la France (Bassin de la Seine, Bassin de la Somme et Nord)". $9^{\mathrm{e}}$ Congrès UISPP, Nice, 1976, 177-180.

SOMMÉ J., 1977 - Les plaines du Nord de la France et leur bordure. Etude géomorphologique. Thèse de Doctorat d'Etat, Paris et Lille, 2 volumes, $810 \mathrm{p}$

SOMMÉ J., 1989 - La terrasse de Grâce. In A. Tuffreau (dir.), Livretguide de l'excursion dans la vallée de la Somme. Colloque international «L'Acheuléen dans l'Ouest de l'Europe», Saint-Riquier, 1989, 34-40.

STREMME H.E., 1985 - Altersbestimmung und Paläoböden in der Quatärstratigraphie. Bulletin de l'Association Française pour l'Étude du Quaternaire, 2-3, 159-166.

STUART A.J., 1975 - The vertebrate fauna of the type Cromerian. Boreas, 4, 63-76.

STUART A.J., 1981 - A comparison of the Middle Pleistocene mammal faunas of Voigtstedt (Thuringia, GDR) and West Runton (Norfolk, England). Quartärpaläontologie, 4, 155-163.

STUART A.J., \& LISTER A.M., 2001 - The mammalian fauna of Pakefield/Kessingland and Corton, Suffolk: evidence for a new temperate episode in the British early Middle Pleistocene. Quaternary Science Reviews, 20, 1677-1692.

TOBIEN H., 1975 - Pleistozäne warmzeiten und Saügetiere in Europa. Quartärpaläontologie, 1, 221-233.

TROFIMOV B.A., 1955 - Questions on the origin and development of the Quaternary mammal fauna in the temperate and northern zone. Trudy Kommission cetv period, 12, 106-120.

TUFFREAU A., MUNAUT A.V., PUISSEGUR J.-J., \& SOMMÉ J., 1981 - Les basses terrasses dans les vallées du Nord de la France et de la Picardie: stratigraphie et Paléolithique. Bulletin de la Société Préhistorique Française, 78 (10-12), 291-305.

TUFFREAU A., 1987 - Le Paléolithique inférieur et moyen du Nord de la France (Nord, Pas-de-Calais, Picardie) dans son cadre stratigraphique. Thèse de Doctorat d'État, Université des Sciences et Techniques Lille-Flandres-Artois, 2 tomes, 609 p.

TUFFREAU A., \& SOMMÉ J. (dir), 1988 - Le gisement paléolithique moyen de Biache-Saint-Vaast (Pas-de-Calais). Volume I. Paris, Mémoire de la Société Préhistorique Française, 21, 338 p.

TUFFREAU A., ANTOINE P., CHASE P. G., DIBBLE H. L., ELLWOOD B. B., KOLFSCHOTEN T. VAN, LAMOTTE A., LAURENT M., MCPHERRON S. P., MOIGNE A.-M., \& MUNAUT A. V., 1995 - Le gisement acheuléen de Cagny-l'Épinette (Somme). Bulletin de la Société Préhistorique Française, 92 (2), p. 169-191.

TUFFREAU A., 2001 - Le gisement de Cagny-la Garenne II (Somme) : introduction. In A. Tuffreau (dir.), L'Acheuléen dans la 
vallée de la Somme: données récentes. Villeneuve d'Ascq, Publications du CERP, 6, 41-43.

TUFFREAU A., ANTOINE P., MARCY J.-L., \& SEGARD N., 2001

- Les industries paléolithiques à nombreux bifaces du mont de l'Évangile à Gentelles (Somme). In D. Cliquet (dir.), Les industries à outils bifaciaux du Paléolithique moyen d'Europe occidentale. ERAUL, Liège, 98, 29-41.

TURNER A., 1992 - Villafranchian-Galerian larger Carnivores of Europe: dispersions and extinctions. In W. von Koenigswald \& L. Werdelin (eds.), Mammalian Migration and Dispersal Events in the European Quaternary. Frankfurt am Main, Courier Forschungsinstitut Senckenberg, 153, 153-160.

VANDERMEERSCH B., 1978 - Étude préliminaire du crâne humain du gisement paléolithique de Biache-Saint-Vaast (Pas-de-Calais) Bulletin de l'Association Française pour l'Étude du Quaternaire, 54-56, 65-67.
VANDERMEERSCH B., 1982 - L'Homme de Biache-Saint-Vaast, comparaison avec l'Homme de Tautavel. In «L'Homo erectus et la place de l'Homme de Tautavel parmi les Hominidés fossiles». prétirage $1^{\text {er }}$ Congrès international de Paléontologie Humaine, Nice, 1982, volume 2, 894-900.

VAN VLIET-LANOË B., CLIQUET D., AUGUSTE P., FOLZ E., KEEN D., SCHWENNINGER J.-L., MERCIER N., ALIX P., ROUPIN Y., MEURISSE M., \& SEIGNAC H., 2006 - L'abri sousroche du Rozel (France, Manche): un habitat de la phase récente du Paléolithique moyen dans son contexte géomorphologique. Quaternaire, 17 (3), 207-258. 